\title{
Improved Technique for Design of Perfect Reconstruction FIR QMF Banks with Lossless Polyphase Matrices
}

\author{
P. P. VAIDYANATHAN, SENIOR MEMBER, IEEE, TRUONG Q. NGUYEN, STUdENT MEMBER, IEEE, \\ ZINNUR DOĞANATA, STUDENT MEMBER, IEEE, AND TAPIO SARAMÄKI
}

\begin{abstract}
This paper develops an improved technique for the design of analysis filters in an $\boldsymbol{M}$ channel maximally decimated FIR perfect reconstruction QMF bank, having a lossless polyphase-component matrix $E(z)$. As in earlier work, the aim is to optimize the parameters characterizing $E(z)$ until the sum of the stopband energies of the analysis filters is minimized. There are four new ingredients in the procedure reported here. The first is a technique for efficient initialization of one of the $M$ analysis filters, as a spectral factor of an $M$ th band filter. This factorization itself is done without root-finding techniques, in an efficient manner using the eigenfilters approach. The second component is the initialization of the internal parameters which characterize $E(z)$, based on the above spectral factor. In earlier work, the parameters characterizing the lossless $E(z)$ were rotation angles, which resulted in slow convergence of the above-mentioned optimization. The third ingredient of the improved approach is a modified characterization, mostly free from rotation angles, of the lossless FIR $E(z)$. The fourth component of improvement is the incorporation of symmetry among the analysis filters, so as to minimize the number of unknown parameters being optimized. The resulting design procedure always gives better filter responses than earlier ones (for a given filter length), and converges much faster.
\end{abstract}

\section{INTRODUCTION}

$\mathrm{I}_{M}^{\mathrm{N}}$ $\mathrm{N}$ this paper we consider the maximally decimated $M$-channel analysis/synthesis system shown in Fig. 1. The basic operational principles of this analysis/synthesis system and its applications are discussed in a number of references [1]-[13]. The analysis filters $H_{k}(z)$ split the signal $x(n)$ into $M$ subband signals which are then decimated by $M$ and encoded prior to transmission. At the synthesis end, the $M$ subband signals are decoded, interpolated, and recombined using the synthesis filters $F_{k}(z)$. Ignoring the nonlinear coding/decoding error and quantization errors in the filter implementations, the signal $\hat{x}(n)$ suffers from three errors [5], viz., aliasing, amplitude distortion, and phase distortion. Several techniques have been discussed in the past for eliminating (partially or completely) some or all of these errors (see [1]-[13]). In this paper we shall restrict our attention to one such technique, and incorporate several improvements.

Manuscript received May 30, 1988; revised November 25, 1988. This work was supported in part by the National Science Foundation under Grants DCI 8552579 and MIP 8604456.

P. P. Vaidyanathan, T. Q. Nguyen, and Z. Doğanata are with the Department of Electrical Engineering, California Institute of Technology, Pasadena, CA 91125.

T. Saramäki is with the Department of Electrical Engineering, Tampere University of Technology, SF-33101 Tampere, Finland.

IEEE Log Number 8928127.

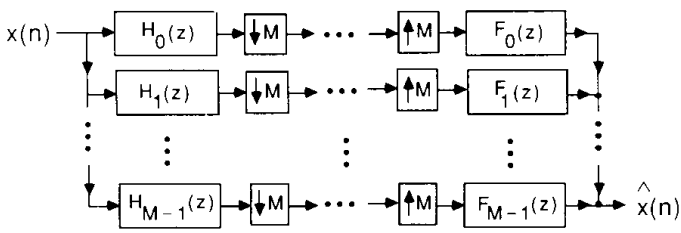

Fig. 1. The $M$-channel maximally decimated QMF bank.

In the literature, two-channel versions of the system of Fig. 1 have been referred to as the quadrature mirror filter (QMF) banks [1]-[4]. This is because the magnitude responses $\left|H_{1}\left(e^{j \omega}\right)\right|$ and $\left|H_{0}\left(e^{j \omega}\right)\right|$ are images of each other with respect to the frequency $\pi / 2$ which is a quarter of the sampling frequency $2 \pi$. For the case of general $M$, the structure of Fig. 1 should not actually be called the QMF bank because the traditional 2-channel meaning does not hold. However, as the word QMF has been used in the past by other authors, we shall retain the same jargon.

The technique we shall discuss here is the one described in [12] (also see [13]). Here, all three distortions mentioned above are eliminated, so that $\hat{x}(n)$ is a delayed version of $x(n)$, i.e., $\hat{x}(n)=c x\left(n-n_{0}\right), c \neq 0$. This is called the perfect reconstruction (abbreviated PR) property, and the QMF bank of Fig. 1 is then said to be a PR system.

The method in [12] is based on the following observation. Each analysis filter $H_{k}(z)$ can be written in the form $H_{k}(z)=\Sigma_{l=0}^{M-1} z^{-l} E_{k l}\left(z^{M}\right)$, and each synthesis filter $F_{k}(z)$ can be written in the form $F_{k}(z)=\Sigma_{l=0}^{M-1}$ $z^{-(M-1-l)} R_{l k}\left(z^{M}\right)$. The quantities $E_{k l}(z), 0 \leq l \leq M-$ 1 are the $M$ polyphase components [14], [2] of the $k$ th analysis filter $H_{k}(z)$. Similarly, $R_{l k}(z)$ are the $M$ polyphase components of $F_{k}(z)$. These components can be used to define two $M \times M$ matrices $E(z)=\left[E_{k l}(z)\right]$ and $\boldsymbol{R}(z)=\left[\boldsymbol{R}_{l k}(z)\right]$, called the polyphase component matrices for the analysis bank and synthesis bank, respectively. With these definitions, the QMF bank of Fig. 1 can always be redrawn as in Fig. 2. The method described in [12] constrains the matrix $\boldsymbol{E}(z)$ to be FIR and lossless (i.e., $\boldsymbol{E}\left(e^{j \omega}\right)$ to be unitary for all $\left.\omega\right)$. Under this condition, if the matrix $\boldsymbol{R}(z)$ is chosen as ${ }^{1} \boldsymbol{R}(z)=\tilde{\boldsymbol{E}}(z)$, then

${ }^{1}$ See the end of this section for meanings of $\boldsymbol{A}^{\dagger}, \tilde{A}$, etc 


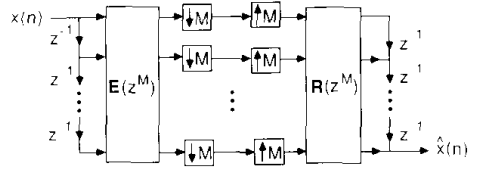

Fig. 2. Redrawing of Fig. 1 in terms of polyphase matrices $\boldsymbol{E}(z)$ and $\boldsymbol{R}(z)$.

the system of Fig. 1 is forced to be a PR system. With lossless $\boldsymbol{E}(z)$, this choice of $\boldsymbol{R}(z)$ is equivalent to choosing the synthesis filters as

$$
F_{k}(z)=z^{-S} \tilde{H}_{k}(z)
$$

so that $f_{k}(n)$ are time-reversed (and conjugated) versions of $h_{k}(n)$. Thus, once the analysis filters $h_{k}(n), 0 \leq k \leq$ $M-1$ are known, we can find $f_{k}(n), 0 \leq k \leq M-1$, of the PR system easily, without matrix inversions. Notice that, with this setup $\left\{F_{k}(z)\right\}$ are also FIR filters of the same lengths as $\left\{H_{k}(z)\right\}$ 's.

The central problem in such a design of a PR QMF bank is therefore the design of analysis filters $H_{k}(z)$ under the constraint that $\boldsymbol{E}(z)$ be lossless. With $\boldsymbol{E}(z)$ constrained to be lossless, the aim is to minimize the sum of stopband energies

$$
\sum_{k=0}^{M-1} \int_{\text {stopband }}\left|H_{k}\left(e^{j \omega}\right)\right|^{2} d \omega
$$

by optimizing the parameters characterizing $\boldsymbol{E}(z)$. The passbands of $H_{k}(z)$ automatically come out to be good for reasons mentioned in [12].

\section{A. Summary of Past Designs}

In the above optimization, the objective function (2) is highly nonlinear with respect to the parameters of $\boldsymbol{E}(z)$. Moreover, no convenient initialization point for the parameters of $\boldsymbol{E}(z)$ was available in the past. Finally, the parameters in $E(z)$ were rotation angles [12], which required the computation of several cosines and sines in order to evaluate (2) for a given parameter set. These facts resulted in very slow convergence of the optimization process. Because of the possibility of multiple minima, it was also necessary to perform optimization with several random initial points. For these reasons, the designs in [12] were restricted to low-order analysis filters. For example, the three channel design in [12] has analysis filters of length 15 , each filter having a stopband attenuation of only about $20 \mathrm{~dB}$.

\section{B. Some Recent Improvements}

It was soon observed [15] that, if the analysis filters were further constrained to have pairwise image property

$$
\left|H_{k}\left(e^{j \omega}\right)\right|=\left|H_{M-1-k}\left(e^{j(\omega-\pi)}\right)\right|
$$

this reduces the number of degrees of freedom [i.e., the number of parameters in $\boldsymbol{E}(z)$ ] for a given filter length, resulting in faster optimization and better filters. Such a design example for $M=3$, with analysis filter lengths equal to 62 , can be found in [15], providing stopband at- tenuations of about $35 \mathrm{~dB}$. The initialization of parameters in [15], however, continued to be random.

Subsequent to this, it was realized that the initialization of parameters can actually be done more judiciously [16]. This is based on several facts. First, if $\boldsymbol{E}(z)$ is lossless, then each analysis filter $H_{k}(z)$ is a spectral factor of an $M$ th band filter (i.e., $\tilde{H}_{k}(z) H_{k}(z)$ are $M$ th band filters ). Second, if any one of the analysis filters, say, $H_{0}(z)$, is fixed at a certain value, then the lossless constraint of $\boldsymbol{E}(z)$ takes away most of the freedom available for the choice of $H_{k}(z), 1 \leq k \leq M-1$. Finally, if $H_{0}(z)$ is somehow initialized, there exists a synthesis procedure to find (i.e., initialize) the majority of parameters of $\boldsymbol{E}(z)$. This leads to a substantial improvement in the design speed, and in the filter performance upon convergence. The design example for $M=3$, reported in [16], is based on this scheme, coupled with the imposition of the symmetry property (3). The analysis filters have length 56 and stopband attenuations exceeding $70 \mathrm{~dB}$.

The final phase of improvement is based on a renewed characterization of an FIR lossless matrix $\boldsymbol{E}(z)$. The earlier characterizations [17], [18] were in the form of a cascade of constant unitary-matrix building blocks, separated by delay elements. The unitary matrices are themselves characterized by rotation angles [17]. Even though this characterization is completely general [18] (in the sense that every lossless FIR matrix can be realized in this form) and canonic (i.e., has the minimum number of delay elements and parameters for a given filter length), the presence of angles makes it necessary to compute several cosines and sines, during each computation of (2). On most general purpose computers, the computation of a cosine (or sine) is about 20 times slower than a multiplication operation. This is, therefore, a major computational overhead, while carrying out the optimization on most machines. A second characterization of FIR lossless matrices was recently outlined [19], which again is general and canonic, but is mostly free from rotation angles. This, coupled with the initialization techniques, and the imposition of symmetry (3), has now emerged into a more efficient algorithm for the design of analysis filters $H_{k}(z)$ of an FIR PR system.

\section{Outline of the Paper}

The purpose of this paper is to give a comprehensive presentation of these results. As many of these improvements have been reported only in conference proceedings [16], [19], [20] (which are incomplete due to space constraints), our presentation here will be self-contained (with the exception that the results in the recent Transactions paper [15] will be freely used). Section II describes our technique to initialize an analysis filter of the PR QMF bank. Section III develops a new minimal characterization for $M \times M$ FIR lossless systems and for $M \times 1$ FIR lossless vectors. Section IV derives the number of degrees of freedom available in the design of an $M \times M$ FIR lossless system, if one row is fixed (corresponding to fixing or initializing an analysis filter). Section $\mathrm{V}$ ties up these im- 
provements with the imposition of pairwise symmetry of analysis filters [15]. A design example is presented in Section VI.

Notations: The downgoing and upgoing arrows in Fig. 1 represent decimators and interpolators as defined in any one of [2], [11]-[13]. Boldfaced italic letters denote matrices. The symbol $I$ denotes the identity matrix, whose dimensions will be clear from the context (if not, a subscript will be used to indicate it). The notations $\boldsymbol{A}^{T}, \boldsymbol{A}^{*}$, and $\boldsymbol{A}^{\dagger}$ denote transposition, conjugation, and transposedconjugation, respectively. For an $M \times 1$ vector $v$, the norm $\sqrt{\boldsymbol{v}^{\dagger} \boldsymbol{v}}$ is denoted as $\|\boldsymbol{v}\|$. A $p \times M$ matrix $A(p \geq$ $M$ ) is said to be orthogonal if $\boldsymbol{A}^{T} \boldsymbol{A}=c \boldsymbol{I}$, and unitary if $\boldsymbol{A}^{\dagger} \boldsymbol{A}=c \boldsymbol{I}$, where $c \neq 0$ is scalar. Subscript $*$ denotes conjugation of coefficients only. Thus, if $E(z)=e_{0}+$ $\boldsymbol{e}_{1} z^{-1}$, then $\boldsymbol{E}_{*}(z)=\boldsymbol{e}_{0}^{*}+\boldsymbol{e}_{1}^{*} z^{-1}$. Finally, $\tilde{\boldsymbol{H}}(z)$ stands for $\boldsymbol{H}_{*}^{T}\left(z^{-1}\right)$. For real-coefficient systems, $\tilde{\boldsymbol{H}}(z)=$ $\boldsymbol{H}^{T}\left(z^{-1}\right)$. In any case, on the unit circle, we have $\tilde{\boldsymbol{H}}(z)$ $=\boldsymbol{H}^{\dagger}(z)$. A $p \times \boldsymbol{M}$ transfer matrix $\boldsymbol{E}(z)$ is said to be lossless if a) its entries $E_{k l}(z)$ are stable and b) $\boldsymbol{E}\left(e^{j \omega}\right)$ is unitary for all $\omega$. For $p=M$, this property is a discretetime version of the well-known property of scattering matrices of LC multiports in electrical network theory [29], [30]. (A lossless matrix with real coefficients is said to be LBR.) Such a system automatically satisfies the property

$$
\tilde{E}(z) \boldsymbol{E}(z)=c \boldsymbol{I} \quad \text { for all } z
$$

where $c \neq 0$ is a constant. For $E(z)$ to be lossless, we require $p \geq M$. If $p=M$, note that (4) is equivalent to $\boldsymbol{E}(z) \tilde{E}(z)=c \boldsymbol{I}$. Note that product of lossless matrices is lossless. The $M$ components of an $M \times 1$ lossless vector are said to be power complementary ( since the magnitude-squares add up to a constant for all $\omega$ ). Note that every column of a lossless system is lossless (hence power complementary).

The degree of a $p \times M$ system (also called McMillan degree [22]) $\boldsymbol{E}(z)$ is equal to the number of scalar delays (i.e., $z^{-1}$ building blocks) required to implement it. Even though it is difficult to find the degree by inspection, it is simple to find in some cases. For example, the degree of an $M \times 1$ FIR transfer matrix $\boldsymbol{H}(z) \triangleq \Sigma_{n=0}^{K} \boldsymbol{h}(n) z^{-n}$ with $\boldsymbol{h}(K) \neq \mathbf{0}$ is equal to $K$. The degree of an $M \times M$ system of the form $z^{-1} \boldsymbol{A}$ is equal to the rank $r$ of $\boldsymbol{A}$ because we can write $z^{-1} \boldsymbol{A}=\boldsymbol{B}\left[z^{-1} \boldsymbol{I}_{r}\right] \boldsymbol{C}$ where $\boldsymbol{B}$ is $M \times$ $r$ and $C$ is $r \times M$.

\section{Initialization of an Analysis Filter}

The set of $M$ analysis filters $H_{k}(z)$, expressed in terms of the polyphase components $E_{k l}(z)$, can be represented as in Fig. 3(a). Since $H_{k}(z)$ are FIR, the entries of $\boldsymbol{E}(z)$ are FIR. In our method, we constrain $\boldsymbol{E}(z)$ to be lossless. The discussions in this section assume $H_{k}(z)$ [hence $\boldsymbol{E}(z)]$ to have real coefficients. A structural representation for $\boldsymbol{E}(z)$, developed in [17] and [18], is shown in Fig. 3(b). This is a cascade of $N$ (real) orthogonal matrices $\boldsymbol{R}_{k}, 0 \leq k \leq N-1$ [where $N-1$ is the degree of

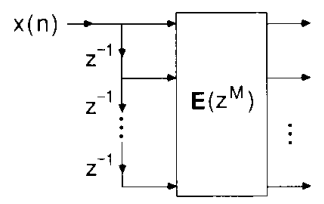

(a)

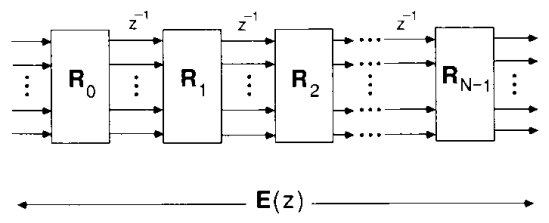

(b)

Fig. 3. Implementation of FIR lossless $E(z)$ as a cascade of unitary matrices separated by delays.

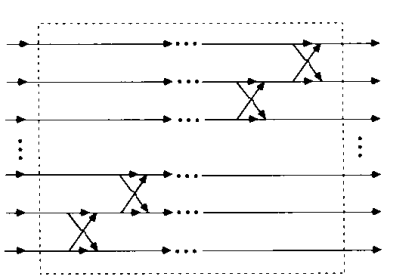

(a)

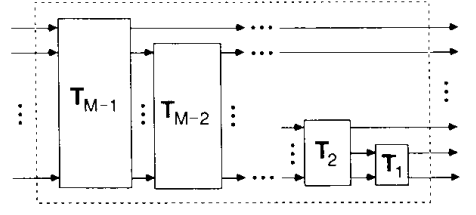

(b)

Fig. 4. Details of the building blocks in Fig. 3(b). In Fig. 4(b), $T_{k}$ is a ( $k$ $+1) \times(k+1)$ matrix with appearance as in Fig. 4(a). The matrix $T_{k}$ has $k$ criss-crosses.

$\boldsymbol{E}(z)$ ] separated by diagonal matrices of the form

$$
\boldsymbol{\Lambda}(z)=\left[\begin{array}{cccc}
z^{-1} & 0 & \cdots & 0 \\
0 & 1 & \cdots & 0 \\
\vdots & \vdots & \ddots & \vdots \\
0 & 0 & \cdots & 1
\end{array}\right]
$$

Recall (Appendix D) that an arbitrary $M \times M$ real orthogonal matrix requires $\left(\begin{array}{c}M \\ 2\end{array}\right)$ real parameters (rotation angles) for complete characterization. The $N-1$ matrices $\boldsymbol{R}_{k}, 0 \leq k \leq N-2$ are special types of orthogonal matrices with $M-1$ planar rotations, as shown in Fig. 4(a). In this figure, each criss-cross represents a planar rotation operation of the form

$$
\left[\begin{array}{cc}
\cos \theta & \sin \theta \\
\sin \theta & -\cos \theta
\end{array}\right] .
$$

The rightmost matrix $\boldsymbol{R}_{N-1}$, on the other hand, is a general orthogonal matrix characterized by $\left(\begin{array}{c}M \\ 2\end{array}\right)$ planar rotations (see Appendix D) as in Fig. 4(b). It is shown in [18] that 
every real-coefficient FIR lossless system $\boldsymbol{E}(z)$ of degree $N-1$ can be represented as in Fig. 3(b) with matrices as in Fig. 4. Conversely, the structure of Fig. 3(b) with matrices as in Fig. 4 necessarily represents a real-coefficient FIR lossless system. Thus, the angles in Fig. 4 form a complete set for characterizing real-coefficient FIR lossless $\boldsymbol{E}(z)$. Moreover, the representation in Fig. 3(b) is canonic (i.e., minimal in number of delays, and in number of planar rotation parameters).

\section{A. The Parameter Space and the Number of Degrees of Freedom}

The total number of planar rotation angles in Fig. 3(b) is

$$
N_{p}=(M-1)(N-1)+\left(\begin{array}{c}
M \\
2
\end{array}\right) .
$$

In order to minimize the objective function (2), it is necessary to optimize these $N_{p}$ parameters. From Fig. 3(b), the maximum length of an analysis filter with this setup is

$$
L^{\prime}=M(N-1)+M
$$

Clearly, the number of parameters $N_{p}$ grows linearly with respect to $L^{\prime}$ and quadratically with respect to $M$. For example, with $M=3$ and analysis filters of length $L^{\prime}=57$, we have $N_{p}=39$. We thus have a large parameter space, and a very nonlinear objective function. With no clue for initialization of angles in Fig. 3(b), the optimization task is formidable indeed.

In Section III we shall show that if we can make an initial guess of one of the length $L^{\prime}$ transfer functions, say $H_{0}(z)$, then almost all the $N_{p}$ parameters can be initialized based only on $H_{0}(z)$. To be more specific, once $H_{0}(z)$ is fixed, only

$$
N_{f}=\left(\begin{array}{c}
M \\
2
\end{array}\right)-(M-1)
$$

parameters are still undetermined in Fig. 3(b). Equation (8) therefore measures the number of degrees of freedom available for the design of $H_{k}(z), 1 \leq k \leq M-1$, once $H_{0}(z)$ has been fixed. This is a consequence of the fact that losslessness of $\boldsymbol{E}(z)$ puts a constraint on $H_{k}(z), k \neq$ 0 , once $H_{0}(z)$ is fixed.

For example, with $M=2$, we have $N_{f}=0$ which shows that we have no freedom of choice of $H_{1}(z)$ once $H_{0}(z)$ is fixed. This is consistent with the earlier observations [5], [12] that, for the $M=2$ case, losslessness of $\boldsymbol{E}(z)$ completely constrains $H_{1}(z)$ to be $H_{1}(z)=$ $z^{-\left(L^{\prime}-1\right)} H_{0}\left(-z^{-1}\right)$. For the $M=3$ case, $N_{f}=1$ so that, once $H_{0}(z)$ is fixed, only one degree of freedom can be exercised in choosing $H_{1}(z)$ and $H_{2}(z)$. It is important to realize that $N_{f}$ is independent of the filter length $L^{\prime}$. Consequently, if $H_{0}(z)$ is known, we have to optimize only the remaining $N_{f}$ parameters, regardless of filter length, to obtain $H_{k}(z), 1 \leq k \leq M-1$. (In practice, we have the option of reoptimizing all the parameters, after ini- tialization of a subset of parameters based on the initial choice of $H_{0}(z)$.)

In order to exploit this to our advantage, it is first necessary to find an appropriate initialization for $H_{0}(z)$, which is the purpose of this section. We can mathematically express Fig. 3(a) as

$$
\boldsymbol{h}(z)=\boldsymbol{E}\left(z^{M}\right) \boldsymbol{d}(z)
$$

where

$$
\boldsymbol{h}(z)=\left[H_{0}(z) H_{1}(z) \cdots H_{M-1}(z)\right]^{T}
$$

and

$$
d(z)=\left[\begin{array}{llll}
1 & z^{-1} \cdots z^{-(M-1)}
\end{array}\right]^{T} .
$$

From the fact that $\boldsymbol{E}(z)$ is lossless, it can be proved (see Appendix A) that the function

$$
G_{k}(z) \triangleq \tilde{H}_{k}(z) H_{k}(z)
$$

is an $M$ th band filter [23], [32], i.e., it satisfies

$$
\sum_{l=0}^{M-1} G_{k}\left(z W^{l}\right)=c
$$

where $W=e^{-j 2 \pi / M}$ and $c$ is a nonzero constant. Conversely, given an arbitrary FIR filter $H_{0}(z)$ such that $G_{0}(z)$ is an $M$ th band filter, there will exist FIR filters $H_{k}(z), 1$ $\leq k \leq M-1$, such that the resulting $\boldsymbol{E}(z)$ is lossless (as we shall see in Section IV).

Thus, one way to initialize $H_{0}(z)$ would be to first design a low-pass $M$ th band filter $G_{0}(z)$ and then compute a spectral factor $H_{0}(z)$ of $G_{0}(z)$. Of course, it is necessary to ensure that $G_{0}\left(e^{j \omega}\right)$ is nonnegative for all $\omega$, so that a spectral factor $H_{0}(z)$ exists. Such an $M$ th band filter can be obtained as follows: first design an $M$ th band equiripple zero-phase FIR filter $G(z)$ as in [23], by using the McClellan-Parks program [24]. Then define $G_{0}(z)=$ $G(z)+\delta$ where $\delta$ is the peak stopband ripple of $G\left(e^{j \omega}\right)$, so that $G_{0}\left(e^{j \omega}\right)$ has all the desired properties. Finally, compute the coefficients of $H_{0}(z)$ such that $G_{0}(z)=$ $H_{0}\left(z^{-1}\right) H_{0}(z)$ (this is the spectral factorization step).

If $H_{0}(z)$ is required to have a stopband attenuation of $70 \mathrm{~dB}$ (as an arbitrary example), then $G_{0}(z)$ has stopband attenuation $140 \mathrm{~dB}$. This implies that $G(z)$ has large order, and has several zeros in the stopband. Under such conditions, the spectral factorization is an inaccurate process, and gives rise to numerical difficulties even when clever techniques such as [25] are employed. In this section we show how $H_{0}(z)$ with the above properties can be directly designed, thereby eliminating the spectral factorization step. According to our experience, this direct approach always gives rise to more accurate and faster designs for the filter $H_{0}(z)$.

B. The Eigenfilter Approach for Initialization of $H_{0}(z)$

Any FIR transfer function $H_{0}(z)$ can be written as

$$
H_{0}(z)=H_{00}(z) H_{01}(z)
$$


where $H_{01}(z)$ has all zeros on the unit circle, and $H_{00}(z)$ has none on the unit circle. If $H_{0}(z)$ is a spectral factor of $G_{0}(z)$, then we can write

$$
G_{0}(z)=G_{00}(z) H_{01}^{2}(z)
$$

where $G_{00}(z)$ has no zeros on the unit circle. The form (15) reflects the fact that zeros of $G_{0}(z)$ on the unit circle must be double so that $G_{0}\left(e^{j \omega}\right)$ is nonnegative for all $\omega$. Since $G_{0}(z)$ has zero phase, $G_{00}(z)$ has zeros occurring in reciprocal pairs.

Our aim is to design $H_{0}(z)$ such that its stopband energy

$$
\int_{\omega s}^{\pi}\left|H_{00}\left(e^{j \omega}\right)\right|^{2}\left|H_{01}\left(e^{j \omega}\right)\right|^{2} d \omega / 2 \pi
$$

is minimized under the constraint that $H_{0}\left(z^{-1}\right) H_{0}(z)$ is an $M$ th band filter. This is equivalent to minimizing the quantity

$$
\varepsilon=\int_{\omega S}^{\pi}\left|G_{00}\left(e^{j \omega}\right)\right|\left|H_{01}\left(e^{j \omega}\right)\right|^{2} d \omega
$$

under the constraint that $G_{0}(z)$ be an $M$ th band filter. The advantage of writing $G_{0}(z)$ in the form (15) and optimizing (17) is that the resulting design will automatically reveal the coefficients of $H_{01}(z)$ and $G_{00}(z)$. If we take $H_{00}(z)$ to be a spectral factor of $G_{00}(z)$, the design of $H_{0}(z)$ is complete. This involves finding only the spectral factor of $G_{00}(z)$ which is a much lower order polynomial than $G_{0}(z)$. In addition, $G_{00}(z)$ has no zeros on the unit circle. It is therefore easy to find a spectral factor $H_{00}(z)$ of $G_{00}(z)$.

In what follows, we shall denote the orders of $H_{00}(z)$ and $H_{01}(z)$ by $l_{0}$ and $l_{1}$, respectively. Accordingly, $G_{00}(z)$ has order $2 l_{0}$ and $G_{0}(z)$ has order $2\left(l_{0}+l_{1}\right)$. Notice that in the above setup $G_{00}(z)$ and $H_{01}(z)$ are linear-phase transfer functions. Assume, for a moment, that the coefficients of $G_{00}(z)$ are known. Given the function $G_{00}(z)$, this task of finding the linear-phase polynomial $H_{01}(z)$ to minimize (17) is precisely the weighted eigenfilter design problem described in [28] (to be elaborated below). While minimizing (17) we shall impose a constraint that avoids the trivial solution $H_{01}(z)=0$.

Now assume that, for a given $G_{00}(z)$, we have found the coefficients of $H_{01}(z)$ using the eigenfilters approach. With $H_{01}(z)$ fixed at this value, we can recompute the coefficients of $G_{00}(z)$ such that the quantity $G_{0}(z)$ in (15) is indeed an $M$ th band filter. With this new $G_{00}(z)$, we can again solve for $H_{01}(z)$ to minimize (17). A few repetitions (typically four or five) result in an $M$ th band filter $G_{0}(z)$ with excellent stopband attenuation. Once $G_{00}(z)$ and $H_{01}(z)$ are found in this manner, it is only necessary to find a spectral factor $H_{00}(z)$ of $G_{00}(z)$. The analysis filter $H_{0}(z)$ is then obtained as in (14). Summarizing, the steps in the design of $H_{0}(z)$ are as follows.

1) Initialize $G_{00}\left(e^{j \omega}\right)$ to be unity for all $\omega$.

2) For this $G_{00}(z)$, find the linear phase transfer function $H_{01}(z)$ of order $l_{1}$ such that (17) is minimized (under appropriate constraints to be elaborated).
3) With $H_{01}(z)$ so fixed, find the linear phase transfer function $G_{00}(z)$ of order $2 l_{0}$ such that the product (15) is an $M$ th band filter.

4) If the result $G_{0}(z)$ is not satisfactory, go to step 2 .

Even though there is no formal proof that this approach converges, excellent designs for $G_{0}(z)$ could be obtained in every case attempted. At most, five iterations between steps 2 and 3 were necessary in all cases. The details of steps 2 and 3 are described next.

The Eigenfilter Approach [28] for Step 2: Let $H_{01}(z)$ be a real-coefficient linear-phase FIR filter [26] of the form

$$
H_{01}(z)=\sum_{n=0}^{l} h_{01}(n) z^{-n}
$$

with

$$
h_{01}(n)=h_{01}\left(l_{1}-n\right) .
$$

Assume first that $l_{1}$ is even and let $M_{1}=l_{1} / 2$. We can express the frequency response as [26], [27, p. 72]

$$
H_{01}\left(e^{j \omega}\right)=e^{-j M_{1} \omega} \sum_{n=0}^{M_{1}} b_{n} \cos (\omega n) .
$$

The coefficients $b_{n}$ are given by $b_{0}=h\left(M_{1}\right)$ and $b_{n}=$ $2 h\left(M_{1}-n\right), n \neq 0$. If we define the vectors

$$
\begin{aligned}
\boldsymbol{b} & =\left[\begin{array}{llll}
b_{0} & b_{1} & \cdots & b_{M_{1}}
\end{array}\right]^{T}, \\
\boldsymbol{c}(\omega) & =\left[\begin{array}{llll}
1 & \cos (\omega) & \cdots & \cos \left(M_{1} \omega\right)
\end{array}\right]^{T}
\end{aligned}
$$

we can express $(20)$ as $H_{01}\left(e^{j \omega}\right)=e^{-j M_{\downarrow} \omega} \boldsymbol{b}^{T} \boldsymbol{c}(\omega)$. Now consider the quantity

$$
\varepsilon=\int_{\omega S}^{\pi} W\left(e^{j \omega}\right)\left|H_{01}\left(e^{j \omega}\right)\right|^{2} d \omega
$$

where $W\left(e^{j \omega}\right)$ is a nonnegative real function of $\omega$. Suppose we wish to minimize (22) under the constraint

$$
\boldsymbol{b}^{T} \boldsymbol{b}=1 \text {. }
$$

We can rewrite $(22)$ as

$$
\varepsilon=\boldsymbol{b}^{T}\left[\int_{\omega S}^{\pi} W\left(e^{j \omega}\right) \boldsymbol{c}(\omega) \boldsymbol{c}^{T}(\omega) d \omega\right] \boldsymbol{b} .
$$

The integral in (24) is an $\left(M_{1}+1\right) \times\left(M_{1}+1\right)$ matrix $\boldsymbol{P}$. We can rewrite $(24)$ as

$$
\mathcal{E}=\boldsymbol{b}^{T} \boldsymbol{P b} \text {. }
$$

Clearly, the matrix $\boldsymbol{P}$, which is real and symmetric, is positive definite (except under trivial situations such as $\omega_{S}=\pi$, or $W\left(e^{j \omega}\right)\left|H_{01}\left(e^{j \omega}\right)\right|^{2}=0$ for all $\left.\omega\right)$. The vector $\boldsymbol{b}$ minimizing (25) under the constraint (23) is the eigenvector of $\boldsymbol{P}$ corresponding to its minimum eigenvalue. Once $\boldsymbol{b}$ is found in this way, the coefficients of $H_{01}(z)$ which minimize (22) can be obtained. For obvious reasons, $H_{01}(z)$, so found, will be called an eigenfilter. It is clear that with $W\left(e^{j \omega}\right)$ taken as $\left|G_{00}\left(e^{j \omega}\right)\right|$, we can find the optimal linear-phase $H_{01}(z)$ using this eigenfilter approach. 
If the order $l_{1}$ is odd, the procedure differs in some minor details, which can be inferred from [28, p. 13].

Details of Step 3: Given a linear-phase transfer function $G_{01}(z) \triangleq H_{01}^{2}(z)$ of order $2 l_{1}$, the task here is to find the coefficients of a linear phase filter $G_{00}(z)$ of order $2 l_{0}$ such that the product $G_{0}(z)$ in (15) is an $M$ th band filter. It is well known [23] that the $M$ th band property (13) of $G_{0}(z)$ is equivalent to the condition

$$
g_{0}(M n)= \begin{cases}\frac{1}{M} & \text { for } n=0 \\ 0 & \text { otherwise. }\end{cases}
$$

With $g_{00}(n)$ and $g_{01}(n)$ denoting the coefficients of $G_{00}(z)$ and $G_{01}(z)$, respectively, we see that the coefficients $g_{0}(n)$ of $G_{0}(z)$ are obtained by convolving $g_{00}(n)$ with the known coefficients $g_{01}(n)$. As a result, the condition (26) gives rise to a set of linear equations from which we can solve for the coefficients $g_{00}(n)$ of $G_{00}(z)$.

To be more specific about the number of equations and variables, recall that the order of $G_{0}(z)$ is $2\left(l_{0}+l_{1}\right)$. Express $\left(l_{0}+l_{1}\right)$ in the form

$$
\left(l_{0}+l_{1}\right)=p_{0} M+p_{1}
$$

where $0 \leq p_{1} \leq M-1$. Then the $M$ th band constraint (26) of $G_{0}(z)$ gives rise to $p_{0}+1$ equations. This implies that we require $p_{0}+1$ independent coefficients in the linear-phase filter $G_{00}(z)$. From this, we deduce that the order of $G_{00}(z)$ should be $2 p_{0}$. In other words, $l_{0}=p_{0}$. This, along with (27a), yields

$$
l_{0}=\frac{l_{1}-p_{1}}{M-1} .
$$

Summarizing, given an arbitrary positive integer $l_{1}$, we pick $p_{1}$ in the range $0 \leq p_{1} \leq M-1$ such that the ratio (27b) is an integer. Clearly, $p_{1}$ and $l_{0}$ come out to be unique. This gives the smallest $l_{0}$ such that $G_{0}(z)$ satisfies (26). We then solve for the coefficients of $g_{00}(n)$ by inverting an approprite $\left(l_{0}+1\right) \times\left(l_{0}+1\right)$ matrix. The details are readily developable and can be found in the paper by Nguyen et al. [20].

\section{New Characterizations for FIR Lossless SYSTEMS}

Recall that $\boldsymbol{E}(z)$ is an $M \times M$ matrix. Its McMillan degree (or simply the degree) is, by definition, the minimum number of delay elements (i.e., $z^{-1}$ elements) required to implement $\boldsymbol{E}(z)$. It is known [17] that any realcoefficient $M \times M$ FIR lossless matrix with degree $N$ 1 can be realized as in Fig. 3(b). As mentioned in Section II, the building blocks in Fig. 3(b) are orthogonal matrices, which in turn are combinations of planar rotations.

In this section we shall obtain a second type of characterization (or realization) of FIR lossless matrices, which are free from planar rotations. We find this characterization to be more convenient for optimization of (2). This realization shares the minimality property of Fig. 3(b) (both in terms of number of delays and number of parameters).

We shall obtain the results for the general case where $\boldsymbol{E}(z)$ has complex coefficients, as it turns out to be no more complicated than the real case. Two crucial properties of FIR lossless systems are required in the developments, and are stated next.

Property 3.1: Let $\boldsymbol{H}(z)$ be an $M \times M$ causal FIR lossless transfer matrix of degree $N-1$. Then

$$
\operatorname{det} \boldsymbol{H}(z)=c z^{-(N-1)}
$$

where $c$ is a nonzero constant. As a result, the degree $N$ -1 of $\boldsymbol{H}(z)$ is equal to the degree of the determinant of $\boldsymbol{H}(z)$ :

$$
\operatorname{deg} \boldsymbol{H}(z)=\operatorname{deg} \operatorname{det} \boldsymbol{H}(z) .
$$

This property, which is not true for arbitrary linear time invariant systems, is true for FIR and IIR lossless systems with complex or real coefficients. For the FIR case with real coefficients, the proof can be seen by noting that the determinant of $\boldsymbol{E}(z)$ is the product of the determinants of the $M \times M$ elements in the cascade of Fig. 3(b), which is clearly of the form $c z^{-(N-1)}$. For the FIR case with complex coefficients, a representation as in Fig. 3(b) is valid with minor modifications (with "complex rotators", taking the place of the real ones). The result (28) again follows. Even though IIR systems are not considered in this paper, we note for completion that in this case det $\boldsymbol{H}(z)=c A(z)$, where $A(z)$ is an allpass function with degree equal to that of $\boldsymbol{H}(z)$.

Property 3.2: Let $\boldsymbol{H}(z)$ be any FIR lossless system with

$$
\boldsymbol{H}(z)=\sum_{n=0}^{K} \boldsymbol{h}(n) z^{-n}
$$

with $\boldsymbol{h}(0) \neq \mathbf{0}$ and $\boldsymbol{h}(K) \neq \mathbf{0}$. Then $\boldsymbol{h}^{\dagger}(0) \boldsymbol{h}(K)=\mathbf{0}$, unless $K=0$. In particular, for the $M \times M$ case, both $\boldsymbol{h}(0)$ and $\boldsymbol{h}(K)$ are singular, and $\boldsymbol{h}(0) \boldsymbol{h}^{\dagger}(K)=\mathbf{0}$ as well.

The proof follows immediately from the paraunitary property $\tilde{\boldsymbol{H}}(z) \boldsymbol{H}(z)=\boldsymbol{I}$ by equating like powers of $z$. The first result we shall derive in this section is the following.

Lemma 3.1: An $M \times M$ causal FIR system $\boldsymbol{H}(z)$ is lossless of degree one if and only if it can be represented in the form

$$
\boldsymbol{H}(z)=\left[\boldsymbol{I}-\boldsymbol{v} \boldsymbol{v}^{\dagger}+z^{-1} \boldsymbol{v} \boldsymbol{v}^{\dagger}\right] \boldsymbol{R}
$$

where $\boldsymbol{R}$ is an arbitrary $M \times M$ unitary matrix and $\boldsymbol{v}$ is an $M \times 1$ column vector with unit norm.

We shall then show that any arbitrary $M \times M$ FIR lossless system of degree $N-1$ can be expressed as a cascade of $N-1$ degree-one systems. To be more specific, we shall prove the following.

Theorem 3.1: Let $\boldsymbol{H}_{N-1}(z)$ be an $M \times M$ causal FIR system. Then, it is lossless of degree $N-1$ if and only if it can be written in the form

$$
\boldsymbol{H}_{N-1}(z)=\boldsymbol{V}_{N-1}(z) \boldsymbol{V}_{N-2}(z) \cdots \boldsymbol{V}_{1}(z) \boldsymbol{H}_{0}
$$


where $\boldsymbol{H}_{0}$ is a constant $M \times M$ unitary matrix, and $\boldsymbol{V}_{k}(z)$ are $M \times M$ degree-one FIR lossless matrices of the form

$$
\boldsymbol{V}_{k}(z)=\left[\boldsymbol{I}-\boldsymbol{v}_{k} \boldsymbol{v}_{k}^{\dagger}+\boldsymbol{v}_{k} \boldsymbol{v}_{k}^{\dagger} z^{-1}\right]
$$

and where $\boldsymbol{v}_{k}$ are $M \times 1$ column vectors of unit norm.

Notice that the parameters characterizing the lossless system now are the $N-1$ unit-norm vectors $\boldsymbol{v}_{k}$ and the unitary matrix $\boldsymbol{H}_{0}$. Matrices of the form $\boldsymbol{v} \boldsymbol{v}^{\dagger}$ appearing in (31) and (33) are diadic matrices. Because of their importance in many of our proofs, Appendix B reviews some properties of diadics. The proof of the above theorem will automatically give rise to a synthesis procedure, which can be used to compute the parameters $\boldsymbol{v}_{k}$ and $\boldsymbol{H}_{0}$ for a given FIR lossless $\boldsymbol{H}(z)$. For the case of lossless systems with real coefficients, we see that the number of parameters in the characterization of $\boldsymbol{H}_{0}$ is $\left(\begin{array}{c}M \\ 2\end{array}\right)$ since it is real orthogonal (Appendix D). Moreover, each of the unitnorm vectors $v_{k}$ has $M-1$ degrees of freedom. The total number of parameters therefore works out to be $N_{p}$ in (6), so that (32) is a minimal characterization. In (32), only $\left(\begin{array}{c}M \\ 2\end{array}\right)$ parameters are planar rotation angles, unlike in Fig. 3(b), where all $N_{p}$ parameters are rotations. We shall use the above form for the polyphase matrix $\boldsymbol{E}(z)$ of Fig. 2 . We shall now prove the above Lemma and Theorem. One proof can be obtained by starting with some of the characterizations in [29] for continuous-time lossless scattering matrices, applying the bilinear transform, and setting all pole locations to $z=0$ (because of the FIR nature). We shall, however, give a self-contained proof, working entirely in the $z$-domain.

Proof of Lemma 3.1: The "if" part can be proved by proving $\boldsymbol{H}(z) \tilde{\boldsymbol{H}}(z)=\boldsymbol{I}$. This follows immediately from (31) by noting that product terms of the form $\boldsymbol{v} \boldsymbol{v}^{\dagger} \boldsymbol{v} \boldsymbol{v}^{\dagger}$ simplify to $\boldsymbol{v} \boldsymbol{v}^{\dagger}$ ( because $\boldsymbol{v}^{\dagger} \boldsymbol{v}=1$ ). To prove the "only if " part, note that any $M \times M$ causal FIR system of degree one can be written as

$$
\boldsymbol{H}(z)=\boldsymbol{h}(0)+\boldsymbol{h}(1) z^{-1}
$$

where $\boldsymbol{h}(0)$ and $\boldsymbol{h}(1)$ are $M \times M$ matrices, with $\boldsymbol{h}(1) \neq$ 0. Note, however, that (34) does not always represent a degree-one matrix. (For example, with $\boldsymbol{h}(1)=\boldsymbol{I}$, the degree of (34) is $M$.) The degree-one condition imposes further restrictions on $\boldsymbol{h}(0)$ and $\boldsymbol{h}(1)$. Thus, from Property 3.2 , both $\boldsymbol{h}(0)$ and $\boldsymbol{h}(1)$ are singular. Moreover (see comment at the end of Section I), the rank of $\boldsymbol{h}(1)$ is required to be equal to unity.

Since $\boldsymbol{H}(z)$ is lossless, we know, in particular, that $\boldsymbol{H}(z)$ is unitary for $z=1$. So we can write

$$
\boldsymbol{H}(z)=\left(1-z^{-1}\right) \boldsymbol{S}+\boldsymbol{R}
$$

where $\boldsymbol{R}$ is a constant unitary matrix. Losslessness of (34) implies, in particular, the condition $\boldsymbol{h}(0) \boldsymbol{h}^{\dagger}(1)=\mathbf{0}$. In view of (35), this condition is equivalent to

$$
R S^{\dagger}=-S S^{\dagger} \text {. }
$$

Substituting (36) in (35) and rearranging, we get the form

$$
\boldsymbol{H}(z)=\left[\boldsymbol{I}-S S^{\dagger}+z^{-1} S S^{\dagger}\right] R \text {. }
$$

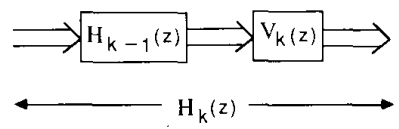

(a)

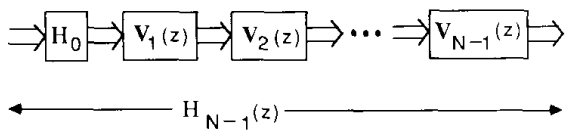

(b)

Fig. 5. Implementation of $M \times M$ lossless FIR $\boldsymbol{H}_{N-1}(z)$ as a cascade of degree-one lossless systems. (a) Degree-reduction step. (b) Overall cascade.

From (37) we see that $\boldsymbol{h}(1)=\boldsymbol{S} \boldsymbol{S}^{\dagger} \boldsymbol{R}$. Since $\boldsymbol{h}$ (1) has rank one, and since $\boldsymbol{R}$ is unitary, $\boldsymbol{S} \boldsymbol{S}^{\dagger}$ has rank one. So we can rewrite

$$
S S^{\dagger}=v v^{\dagger}
$$

where $v$ is an $M \times 1$ column vector. We therefore arrive at the form (31) for $\boldsymbol{H}(z)$. It remains to be shown that $\boldsymbol{v}$ has unit norm. For this, note that the quantity inside the square brackets in (31), which is $\boldsymbol{H}(z) \boldsymbol{R}^{\dagger}$, is lossless (in particular, unitary for $z=-1$ ) so that $I-2 v v^{\dagger}$ is unitary. From Appendix B (property 3) it follows that $v$ should have unit norm. This completes the proof of Lemma 3.1.

Proof of Theorem 3.1: The "if", part follows trivially because (32) is a product of lossless systems and is hence lossless. Consider next the "only if " part. Suppose $\boldsymbol{H}_{k}(z)$ is an $M \times M$ causal FIR lossless system with degree $k$. Let the impulse response coefficients be $\boldsymbol{h}_{k}(n)$ so that $\boldsymbol{H}_{k}(z)=\sum_{n=0}^{k} \boldsymbol{h}_{k}(n) z^{-n}$. (Notice that $\boldsymbol{h}_{k}(k)$ could be null even though $\boldsymbol{H}_{k}(z)$ has degree $k$.) We will show that it can be realized as

$$
\boldsymbol{H}_{k}(z)=\left[\boldsymbol{I}-\boldsymbol{v}_{k} \boldsymbol{v}_{k}^{\dagger}+\boldsymbol{v}_{k} \boldsymbol{v}_{k}^{\dagger} z^{-1}\right] \boldsymbol{H}_{k-1}(z),
$$

i.e., as in Fig. 5(a), where

1) The quantity $\boldsymbol{v}_{k}$ is an appropriate $M \times 1$ column vector with unit norm, and

2) $\boldsymbol{H}_{k-1}(z)$ is an $M \times M$ causal FIR lossless system of degree $k-1$.

This step is equivalent to extracting a lossless system

$$
\boldsymbol{V}_{k}(z)=\left[\boldsymbol{I}-\boldsymbol{v}_{k} \boldsymbol{v}_{k}^{\dagger}+\boldsymbol{v}_{k} \boldsymbol{v}_{k}^{\dagger} z^{-1}\right]
$$

of degree one from $\boldsymbol{H}_{k}(z)$ to obtain the reduced degree lossless system $\boldsymbol{H}_{k-1}(z)$. The system $\boldsymbol{H}_{k-1}(z)$ will be called the remainder of the extraction process. If we begin with $k=N-1$ and repeat this extraction process a finite number of times, we obtain the representation of (32) [i.e., the structure of Fig. 5(b)], for $\boldsymbol{H}_{N-1}(z)$. The final remainder $\boldsymbol{H}_{0}(z)$ is a zero-degree lossless system (i.e., constant unitary matrix), and is denoted by $\boldsymbol{H}_{0}$.

Given a causal FIR $M \times M$ lossless $\boldsymbol{H}_{k}(z)$ with degree $k$, it only remains to show how to construct $\boldsymbol{v}_{k}$ of unit norm, such that $\boldsymbol{H}_{k-1}(z)$ is a causal FIR $M \times M$ lossless matrix of degree $k-1$. With $\boldsymbol{v}_{k}$ restricted to have unit 
norm, $V_{k}(z)$ in (40) is clearly lossless (by Lemma 3.1), so that $V_{k}^{-1}(z)=\tilde{V}(z)$. As a result, (39) is equivalent to

$$
\left[\boldsymbol{I}-\boldsymbol{v}_{k} \boldsymbol{v}_{k}^{\dagger}+\boldsymbol{v}_{k} \boldsymbol{v}_{k}^{\dagger} z\right] \boldsymbol{H}_{k}(z)=\boldsymbol{H}_{k-1}(z) \text {. }
$$

From (41) we see that $\boldsymbol{H}_{k-1}(z)$ is FIR for FIR $\boldsymbol{H}_{k}(z)$. Since $\boldsymbol{H}_{k}(z)$ is also causal, we see that $\boldsymbol{H}_{k-1}(z)$ is causal if and only if $\boldsymbol{v}_{k}$ is such that

$$
\boldsymbol{v}_{k}^{\dagger} \boldsymbol{h}_{k}(0)=\mathbf{0}
$$

where $\boldsymbol{h}_{k}(0)$ is the 0 th impulse response coefficient of $\boldsymbol{H}_{k}(z)$. The losslessness of $\boldsymbol{H}_{k}(z)$ ensures that $\boldsymbol{h}_{k}(0)$ is singular (Property 3.2), so that there exists a unit-norm column vector $\boldsymbol{v}_{k}$ satisfying (42). Assume that $\boldsymbol{v}_{k}$ has been so chosen. Since $\boldsymbol{V}_{k}(z)$ and $\boldsymbol{H}_{k}(z)$ are paraunitary, it is clear from (41) that $\boldsymbol{H}_{k-1}(z)$ is paraunitary. In summary, $\boldsymbol{H}_{k-1}(z)$ is a causal lossless FIR system. It only remains to show that its degree is $k-1$. For this, we invoke Property 3.1 , which says that for $M \times M$ causal FIR lossless systems, the determinant has the form (28) where $N-1$ is the degree. Accordingly, we have det $\boldsymbol{H}_{k}(z)=c_{1} z^{-k}$, and det $\boldsymbol{V}_{k}(z)=c_{2} z^{-1}$. Taking determinants on both sides of (41), we immediately see that det $\boldsymbol{H}_{k-1}(z)=c z^{-(k-1)}$, so that the degree of $\boldsymbol{H}_{k-1}(z)$ is $k-1$ indeed. This completes the proof of Theorem 3.1.

Theorem 3.1 gives us the most general form of $M \times M$ causal FIR lossless system. This form can therefore be used in place of $\boldsymbol{E}(z)$ in Fig. 3(a). The elements of $\boldsymbol{v}_{k}$ and $\boldsymbol{H}_{0}$ are then optimized to minimize the objective function (2). In this way, we are guaranteed that the search will be conducted over the set of all FIR lossless systems of a given degree. Even though this property was shared by the method in [17], we find that the method just described is more convenient and leads to faster convergence.

As mentioned in Section II, it is of great interest to be able to initialize one of the analysis filters, say $H_{0}(z)$. This is equivalent to initializing one column of $\boldsymbol{E}^{T}(z)$ and seeking to find the remaining columns such that $\boldsymbol{E}^{T}(z)$ is lossless and (2) is minimized. In the next section, details of this will be presented. In preparation for this, we now derive the most general form of $M \times 1$ causal FIR lossless vectors.

Consider an $M \times 1$ column vector $\boldsymbol{P}_{N-1}(z)$ of the form

$$
\boldsymbol{P}_{N-1}(z)=\sum_{n=0}^{N-1} \boldsymbol{p}_{N-1}(n) z^{-n}
$$

where $p_{N-1}(n)$ are $M \times 1$ constants, with $p_{N-1}(N-1)$ $\neq 0$. Clearly, $\boldsymbol{P}_{N-1}(z)$ can be implemented with $N-1$ scalar delays so that its degree is $N-1$. We say that $\boldsymbol{P}_{N-1}(z)$ is lossless if $\tilde{\boldsymbol{P}}_{N-1}(z) \boldsymbol{P}_{N-1}(z)=1$ for all $z$. This is equivalent to the power-complementary property of the $M-1$ components of $\boldsymbol{P}_{N-1}(z)$. The following result will be used in the next section.

Theorem 3.2: Let $\boldsymbol{P}_{N-1}(z)$ be an $M \times 1$ causal FIR system. Then it is lossless of degree $N-1$ if and only if it can be written in the form

$$
\boldsymbol{P}_{N-1}(z)=\boldsymbol{U}_{N-1}(z) \boldsymbol{U}_{N-2}(z) \cdots \boldsymbol{U}_{1}(z) \boldsymbol{P}_{0}
$$

where $\boldsymbol{P}_{0}$ is a constant $M \times 1$ vector of unit norm, and $\boldsymbol{U}_{k}(z)$ are $M \times M$ degree-one FIR lossless matrices of the form

$$
\boldsymbol{U}_{k}(z)=\left[\boldsymbol{I}-\boldsymbol{u}_{k} \boldsymbol{u}_{k}^{\dagger}+\boldsymbol{u}_{k} \boldsymbol{u}_{k}^{\dagger} z^{-1}\right],
$$

where $\boldsymbol{u}_{k}$ are $M \times 1$ unit-norm vectors.

The proof of the above Theorem is again based on a repeated application of the degree-reduction step. There is, however, a fundamental difference because determinants are not meaningful anymore. Given a causal FIR $M$ $\times 1$ lossless system $\boldsymbol{P}_{k}(z)$ of degree $k \neq 0$, the degreereduction step seeks to generate a remainder $\boldsymbol{P}_{k-1}(z)$ such that it is a lower degree causal FIR $M \times 1$ lossless system. This is done by attempting to express $\boldsymbol{P}_{k}(z)$ as

$$
\boldsymbol{P}_{k}(z)=\left[\boldsymbol{I}-\boldsymbol{u}_{k} \boldsymbol{u}_{k}^{\dagger}+z^{-1} \boldsymbol{u}_{k} \boldsymbol{u}_{k}^{\dagger}\right] \boldsymbol{P}_{k-1}(z) \text {. }
$$

The choice of $\boldsymbol{u}_{k}$ is crucial in the degree reduction process. Since $\boldsymbol{u}_{k}$ has unit norm, (45) is lossless so that (46) can be rewritten as

$$
\boldsymbol{P}_{k-1}(z)=\left[\boldsymbol{I}-\boldsymbol{u}_{k} \boldsymbol{u}_{k}^{\dagger}+z \boldsymbol{u}_{k} \boldsymbol{u}_{k}^{\dagger}\right] \boldsymbol{P}_{k}(z)
$$

The remainder function $\boldsymbol{P}_{k-1}(z)$ is causal if and only if $\boldsymbol{u}_{k}^{\dagger} \boldsymbol{p}_{k}(0)=0$. Since $\boldsymbol{p}_{k}(0)$ is a column vector, there exist many unit-norm vectors $\boldsymbol{u}_{k}$ satisfying this condition. In particular, the following choice:

$$
\boldsymbol{u}_{k}=\frac{\boldsymbol{p}_{k}(k)}{\left\|\boldsymbol{p}_{k}(k)\right\|}
$$

works because, due to losslessness of $\boldsymbol{P}_{k}(z)$, the condition $\boldsymbol{p}_{k}^{\dagger}(k) \boldsymbol{p}_{k}(0)=0$ holds (Property 3.2). With $\boldsymbol{u}_{k}$ so chosen, the coefficient of $z^{-k}$ in (47) becomes

$$
\begin{aligned}
{\left[\boldsymbol{I}-\boldsymbol{u}_{k} \boldsymbol{u}_{k}^{\dagger}\right] \boldsymbol{p}_{k}(k) } & =\left\|\boldsymbol{p}_{k}\right\|\left[\boldsymbol{I}-\boldsymbol{u}_{k} \boldsymbol{u}_{k}^{\dagger}\right] \boldsymbol{u}_{k} \\
& =\left\|\boldsymbol{p}_{k}\right\|\left[\boldsymbol{u}_{k}-\boldsymbol{u}_{k}\right]=\mathbf{0}
\end{aligned}
$$

which proves that $\boldsymbol{P}_{k-1}(z)$ has degree $k-1$. In summary, the choice of $\boldsymbol{u}_{k}$ according to (48) ensures that $\boldsymbol{P}_{k-1}(z)$ is a causal $M \times 1$ lossless system of degree $k-1$. Repeated application of this step results in the form (44), proving the theorem.

One consequence of Theorem 3.1 is that a causal $M \times$ $M$ FIR lossless system $\boldsymbol{H}_{N-1}(z)$ of degree $N-1$ can be realized as in Fig. 6(a) where $\boldsymbol{H}_{0}$ is $M \times M$ unitary and $V(z)$ is a special form of $M \times M$ lossless system of degree $N-1$, viz.,

$$
\boldsymbol{V}(z)=\boldsymbol{V}_{N-1}(z) \boldsymbol{V}_{N-2}(z) \cdots \boldsymbol{V}_{1}(z)
$$

with $V_{k}(z)$ as in (40). Similarly, Theorem 3.2 says that a causal $M \times 1$ FIR lossless system $\boldsymbol{P}_{N-1}(z)$ of degree $N$ - 1 can be realized as in Fig. 6(b) where $P_{0}$ is a unitnorm vector and where $\boldsymbol{U}(z)$ is an $M \times M$ lossless system of degree $N-1$, with exactly the same form as $V(z)$, i.e.,

$$
\boldsymbol{U}(z)=\boldsymbol{U}_{N-1}(z) \boldsymbol{U}_{N-2}(z) \cdots \boldsymbol{U}_{1}(z)
$$

Both realizations of Fig. 6 are clearly minimal in delays.

Uniqueness of the Factorization of $\boldsymbol{P}_{N-1}(z)$ : Except for a scale factor of unit magnitude, (48) is the only choice 


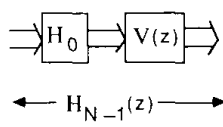

(a)

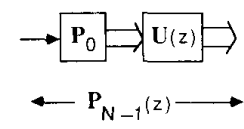

(b)
Fig. 6. Overall representation of the factorization of $\boldsymbol{H}_{N-1}(z)$ and $\boldsymbol{P}_{N-1}(z)$.

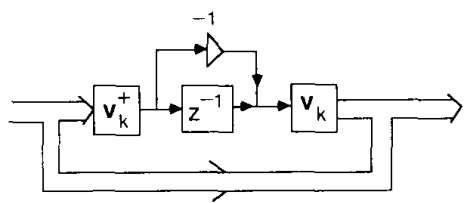

Fig. 7. Implementation of $V_{k}(z)$ using one scalar delay and $2 M$ scalar multipliers

that results in a reduced order causal lossless system $\boldsymbol{P}_{k-1}$. This is because $\left[\boldsymbol{I}-\boldsymbol{u}_{k} \boldsymbol{u}_{k}^{\dagger}\right]$ has rank $\boldsymbol{M}-1$, so that there is precisely one vector $\boldsymbol{u}$ (except for a scale factor) such that $\left[\boldsymbol{I}-\boldsymbol{u}_{k} \boldsymbol{u}_{k}^{\dagger}\right] \boldsymbol{u}=\mathbf{0}$. Since the choice $\boldsymbol{u}=\boldsymbol{c} \boldsymbol{u}_{k}$ works, it is the only possibility. The scale factor $c$ of unit magnitude disappears in the expression $\boldsymbol{u}_{k} \boldsymbol{u}_{k}^{\dagger}$ so that $\boldsymbol{U}_{k}(z)$, and hence $U(z)$, are unique for a given $\boldsymbol{P}_{N-1}(z)$. From (44) we see that $\boldsymbol{P}_{N-1}(1)=\boldsymbol{P}_{0}$, so that the vector $\boldsymbol{P}_{0}$ is unique as well.

Nonuniqueness of the Factorization of $\boldsymbol{H}_{N-1}(z)$ : Notice that the choice of $\boldsymbol{v}_{k}$ according to (42) is necessary and sufficient to obtain a lower degree causal lossless system $\boldsymbol{H}_{k-1}(z)$ from $\boldsymbol{H}_{k}(z)$. Unless the rank of $\boldsymbol{h}_{k}(0)$ is precisely $M-1$, the choice of $\boldsymbol{v}_{k}$ is not unique, so that $\boldsymbol{V}_{k}(z)$ in (33) are not unique. So the realization of $\boldsymbol{H}_{N-1}(z)$ as in Fig. 5(b) is, in general, nonunique. However, from (32) we see that $\boldsymbol{H}_{N-1}(1)=\boldsymbol{H}_{0}$, so that $\boldsymbol{H}_{0}$ is unique. This implies that $\boldsymbol{V}(z)$, which is equal to $\boldsymbol{H}_{N-1}(z) \boldsymbol{H}_{0}^{-1}$, is unique. Summarizing, an $M \times M$ causal FIR lossless system of degree $N-1$ can be factorized as $\boldsymbol{H}_{N-1}(z)=$ $\boldsymbol{V}(z) \boldsymbol{H}_{0}$, where $\boldsymbol{H}_{0}$ and $\boldsymbol{V}(z)$ are unique, even though the building blocks $V_{k}(z)$ may be nonunique.

Implementation of (33) with Minimum Number of Delays: It is possible to implement each building block of the form (33) with only one delay because the degree of $V_{k}(z)$ is unity. Fig. 7 shows an implementation of $V_{k}(z)$ with one scalar delay and $2 M$ multipliers. A cascade of building blocks of this form, terminated on the left with $\boldsymbol{H}_{0}$, completes the minimal implementation of (32).

\section{The Degree of Freedom After Initialization}

Assume that we have designed a causal FIR filter $H_{0}(z)$ as in Section II, so that it is a spectral factor of an $M$ th band filter $G_{0}(z)$. This completely determines the 0 th row

$$
\boldsymbol{e}_{0}^{T}(z)=\left[\begin{array}{llll}
E_{00}(z) & E_{01}(z) & \cdots & E_{0, M-1}(z)
\end{array}\right]
$$

of $\boldsymbol{E}(z)$. Because of the $M$ th band property of $G_{0}(z)$, the $M \times 1$ column-vector $e_{0}(z)$ turns out to be lossless (Appendix A). Let $N-1$ denote the degree of $e_{0}(z)$. We know from Theorem 3.2 that $e_{0}(z)$ can be represented as

$$
\boldsymbol{e}_{0}(z)=U(z) \boldsymbol{P}_{0}
$$

where $U(z)$ is as in (50), and $\boldsymbol{P}_{0}$ is an $M \times 1$ unit-norm vector. If we construct an $M \times M$ unitary matrix

$$
\boldsymbol{H}_{0}=\left[\begin{array}{llll}
\boldsymbol{P}_{0} & \boldsymbol{P}_{1} & \cdots & \boldsymbol{P}_{M-1}
\end{array}\right]
$$

with 0th column equal to $\boldsymbol{P}_{0}$ (say, by using Gram-Schmidt procedure), then the $M \times M$ FIR system

$$
\boldsymbol{S}(z)=\boldsymbol{U}(z) \boldsymbol{H}_{0}
$$

is clearly lossless with degree $N-1$. We can now define the lossless polyphase matrix as $\boldsymbol{E}(z) \triangleq \boldsymbol{S}^{T}(z)$ and obtain the FIR analysis filters $H_{k}(z)$ according to Fig. 3(a). This shows that once a spectral factor $H_{0}(z)$ of an $M$ th band filter $G_{0}(z)$ of arbitrary order is obtained, it is easy to find a set of analysis filters $H_{k}(z), 1 \leq k \leq M-1$ of the perfect-reconstruction system. The number of freedoms that can be exercised in the above construction of $H_{k}(z)$, $1 \leq k \leq M-1$, is equal to the number of freedoms available in constructing an $M \times M$ unitary matrix, whose 0 th column is fixed. This number is given by

$$
N_{f}^{\prime}=M^{2}-2 M+1
$$

if the filter coefficients (and hence $\boldsymbol{H}_{0}$ ) are complex, whereas if the coefficients are real, this number is precisely equal to $N_{f}$ given in (8) (see Appendix D for details).

Given the FIR filter $H_{0}(z)$, does this technique cover every possible set of causal FIR filters $H_{k}(z), 1 \leq k \leq$ $M-1$, such that $\boldsymbol{E}(z)$ is lossless? In general, the answer is $n o$, as demonstrated by the following counterexample: consider the trivial transfer function $H_{0}(z)=1$; then

$$
\boldsymbol{e}_{0}(z)=\left[\begin{array}{llll}
1 & 0 & \cdots & 0
\end{array}\right]^{T}
$$

so that we have $U(z)=I$ in (52). This means that $H_{k}(z)$, $1 \leq k \leq M-1$ have degrees equal to 0 . Evidently, this does not cover the PR system [13] given by

$$
\begin{aligned}
& H_{k}(z)=z^{-k}, \\
& F_{k}(z)=z^{-(M-1-k)}, \quad 0 \leq k \leq M-1,
\end{aligned}
$$

for which $E(z)=I$ (which is lossless).

According to Theorem 4.1, which we shall see next, the answer to the above question is yes, provided the degree of $H_{0}(z)$ is equal to the maximum possible degree it can have, based on the implementation of Fig. 3 (i.e., degree of $\left.H_{0}(z)=M(N-1)+M-1\right)$. Since this is a reasonable assumption, in practice, we shall assume so for the rest of the paper. This is equivalent to the statement that the degree of $\boldsymbol{e}_{0}(z)$ is equal to that of $\boldsymbol{E}(z)$. The main result to be proved is the following.

Theorem 4.1: Let $\boldsymbol{H}_{N-1}(z)$ be any $M \times M$ causal FIR lossless system with degree $N-1$, whose 0th column $\boldsymbol{P}_{N-1}(z)$ has degree $N-1$. Let (44) be the unique factorization of $\boldsymbol{P}_{N-1}(z)$ where $\boldsymbol{U}_{k}(z)$ are as in (45) with unitnorm $\boldsymbol{u}_{k}$ 's. Let (32) be an arbitrary factorization of $\boldsymbol{H}_{N-1}(z)$ with $\boldsymbol{V}_{k}(z)$ as in (33) with unit-norm $\boldsymbol{v}_{k}$ 's. Then

$$
V_{k}(z)=U_{k}(z), \quad 1 \leq k \leq N-1,
$$

and $\boldsymbol{P}_{0}$ is the 0th column of $\boldsymbol{H}_{0}$. 
Proof: Since the degrees of $\boldsymbol{P}_{N-1}(z)$ and $\boldsymbol{H}_{N-1}(z)$ are both equal to $N-1$, they have realizations of the forms (44) and (32), respectively, with $\boldsymbol{v}_{k} \neq \mathbf{0}$ and $\boldsymbol{u}_{k} \neq \mathbf{0}$ for any $k$. This implies, in particular, that $\boldsymbol{P}_{N-1}(z)$ can be written in two forms, viz. (44), and the following:

$$
\boldsymbol{P}_{N-1}(z)=\boldsymbol{V}_{N-1}(z) \boldsymbol{V}_{N-2}(z) \cdots \boldsymbol{V}_{1}(z) \boldsymbol{H}_{00}
$$

where $\boldsymbol{H}_{00}$ is the 0 th column of $\boldsymbol{H}_{0}$. By uniqueness of the factorization for $\boldsymbol{P}_{N-1}(z)$ (see the end of Section III), the relation (58) immediately follows. Moreover, by uniqueness of $\boldsymbol{P}_{0}$, it is clear that we have $\boldsymbol{P}_{0}=\boldsymbol{H}_{00}$. This completes the proof.

The theorem obviously holds if the phrase " 0 th column" is replaced with "any column.", The consequence is that if any column $\boldsymbol{P}_{N-1}(z)$ of an $M \times M$ causal FIR lossless system $\boldsymbol{H}_{N-1}(z)$ of degree $N-1$ has degree $N-$ 1 , then we have to synthesize (or factorize) only this column, in order to obtain the factorization of the entire $\boldsymbol{H}_{N-1}(z)$. The factorization of this column $\boldsymbol{P}_{N-1}(z)$ places in evidence the degree-one building blocks $V_{k}(z), 1 \leq k$ $\leq N-1$. The terminating matrix $\boldsymbol{H}_{0}$ is evaluated as $\boldsymbol{H}_{0}$ $=\boldsymbol{H}_{N-1}(1)$, completing the factorization of $\boldsymbol{H}_{N-1}(z)$. It is clear that if the column $\boldsymbol{P}_{N-1}(z)$ of degree $N-1$ is fixed a priori, the number of degrees of freedom available in the choice of the remaining columns of $\boldsymbol{H}_{N-1}(z)$ is equal to the number of freedoms available in choosing the unitary matrix $\boldsymbol{H}_{0}$, one of whose columns is fixed.

Recall from Section III that the factorization of $\boldsymbol{H}_{N-1}(z)$ is in general not unique, because the choice of $\boldsymbol{v}_{k}$ satisfying (42) is not unique unless the rank of $\boldsymbol{h}_{k}(0)$ is precisely $M-1$. The above theorem, on the other hand, implies that the factorization of $\boldsymbol{H}_{N-1}(z)$ is indeed unique if one of the columns has the full degree $N-1$. The corollary is that whenever there exists a column of $\boldsymbol{H}_{N-1}(z)$ which has degree $N-1$, the 0th coefficient $\boldsymbol{h}_{N-1}(0)$ of $\boldsymbol{H}_{N-1}(z)$ must have rank $\boldsymbol{M}-1$. A second proof of this fact, which removes the mystery associated with such a coincidence, is given in Appendix C.

\section{Imposing Pairwise Symmetry on the Analysis FILTERS}

The final phase of improvement in our design of perfect-reconstruction FIR QMF banks is the recognition that, in many applications, the responses of the analysis filters $H_{k}(z)$ and $H_{M-1-k}(z)$ are not arbitrary. To be specific, it is often acceptable to let $\left|H_{M-1-k}\left(e^{j \omega}\right)\right|$ be an image of $\left|H_{k}\left(e^{j \omega}\right)\right|$ with respect to $\omega=\pi / 2$. We can exploit this to our advantage for the reason that if $H_{k}(z)$ and $H_{M-1-k}(z)$ are related in this manner, then the number of independent elements in the parameterization of the polyphase matrix $\boldsymbol{E}(z)$ is reduced almost by a factor of two. This feature, combined with the initialization scheme described in Section II, results in an improved design procedure for $H_{k}(z), 0 \leq k \leq M-1$.

The appropriate step now would be to identify a structural form for $E(z)$ so that it will reflect the symmetry of pairs of analysis filters, while at the same time being lossless. Two such structures are presented in considerable

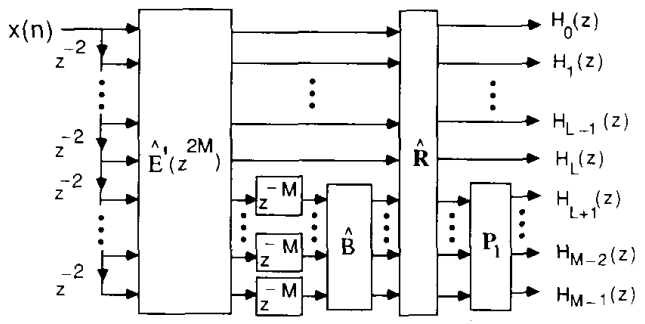

Fig. 8. An FIR analysis bank structure which imposes the relation (62) while retaining $E(z)$ to be lossless.

details in [15] - one for odd $M$ and the other for even $M$. As in [15], the filter coefficients are assumed to be real. The design example to be presented in the next section pertains to odd $M$, so we shall restrict our discussions to odd $M$ here.

Fig. 8 is a reproduction of Fig. 9 in [15]. Here $L=(M$ $-1) / 2$ and $\boldsymbol{E}^{\prime}(z)$ is an FIR lossless matrix with real coefficients. The matrix $\hat{\boldsymbol{B}}$ is $L \times L$ real orthogonal and $\boldsymbol{P}_{1}$ is the permutation matrix

$$
\boldsymbol{P}_{1}=\left[\begin{array}{ccccc}
0 & 0 & \cdots & 0 & 1 \\
0 & 0 & \cdots & 1 & 0 \\
\vdots & \vdots & \ddots & \vdots & \vdots \\
1 & 0 & \cdots & 0 & 0
\end{array}\right]
$$

Finally, $\hat{\boldsymbol{R}}$ is given by

$$
\hat{\boldsymbol{R}}=\left[\begin{array}{llc}
\frac{1}{\sqrt{2}} I_{L} & 0 & \frac{1}{\sqrt{2}} \boldsymbol{I}_{L} \\
\mathbf{0} & 1 & \mathbf{0} \\
\frac{1}{\sqrt{2}} \boldsymbol{I}_{L} & 0 & -\frac{1}{\sqrt{2}} \boldsymbol{I}_{L}
\end{array}\right] .
$$

It is shown in [15] that under this condition, the conventional polyphase matrix $\boldsymbol{E}(z)$ defined according to Fig. 3 (a) is guaranteed to be lossless, and that the analysis filters are automatically related by

$$
H_{k}(z)=H_{M-1-k}(-z) .
$$

The FIR synthesis bank which results in perfect reconstruction can be obtained in the usual way [i.e., by using (1)].

The FIR lossless matrix $\hat{\boldsymbol{E}}^{\prime}(z)$ in Fig. 8 contains the majority of parameters to be optimized during the design of analysis filters. (The matrix $\hat{\boldsymbol{B}}$ has only $\left(\begin{array}{l}L \\ 2\end{array}\right)$ parameters.) Comparing the structure of Fig. 8 to the conventional structure of Fig. 3(a), we see that for a given order of the analysis filters, the number of parameters in $\hat{E}^{\prime}(z)$ is about half the number in $\boldsymbol{E}(z)$ because of the appearance of $z^{2 M}$ rather than $z^{M}$ in Fig. 8. This is precisely due to the fact that the symmetry of $(62)$ is structurally imposed by Fig. 8. Consequently, in minimizing (2), fewer parameters need to be optimized than if Fig. 3(a) were directly used. Moreover, due to the relation (62), the ob- 
jective function (2) can be simplified to have only $L+1$ terms.

Initialization: It is important to adapt the initialization scheme, described in Section II, to the more efficient structure of Fig. 8. Notice that, in view of (62), the transfer function $H_{L}(z)$ is its own image, i.e., $H_{L}(z)=$ $H_{L}(-z)$. In other words, $H_{L}(z)$ has the form $H_{L}^{\prime}\left(z^{2}\right)$. It turns out to be more convenient to initialize this transfer function rather than $H_{0}(z)$. The first step would therefore be to design $H_{L}(z)$ as a bandpass filter centered around $\pi / 2$. This is equivalent to designing a low-pass filter of half the order and replacing $z$ with $-z^{2}$. This can be done by using the eigenfilters approach of Section II. Once $H_{L}(z)$ is known, the $L$ th row $\hat{\boldsymbol{e}}_{L}^{T}(z)$ of the matrix $\hat{\boldsymbol{E}}^{\prime}(z)$ is completely known, because of the form (61) of $\hat{\boldsymbol{R}}$ in Fig. 8 . By using the technique of Section III, we can synthesize $\hat{e}_{L}(z)$ as

$$
\hat{\boldsymbol{e}}_{L}(z)=\boldsymbol{U}(z) \boldsymbol{P}_{0}
$$

where $U(z)$ is the special lossless matrix (50) (but with real coefficients). The complete lossless matrix $\hat{E}^{\prime}(z)$ is then given by

$$
\hat{\boldsymbol{E}}^{\prime}(z)=\boldsymbol{U}(z) \boldsymbol{H}_{0}
$$

Here $\boldsymbol{H}_{0}$ is a real constant orthogonal matrix whose $L$ th column is $\boldsymbol{P}_{0}$, and whose remaining columns are free. This gives us $N_{f}$ degrees of freedom [(8)] in the structure. These parameters, and the $\left(\begin{array}{l}L \\ 2\end{array}\right)$ parameters in the choice of $\hat{\boldsymbol{B}}$, are available as free parameters in the design of the remaining $M-1$ analysis filters. In practice, these parameters can be randomly initialized and optimized. In fact, the parameters which were initialized because of the knowledge of $H_{L}(z)$ can also be reoptimized so that all the $M$ filters come out to be good. Further details will be presented along with the design example in the next section.

It should be noted that in the structure of Fig. 8, the lengths of the filters $h_{k}(n), k \neq L$ are typically higher than $h_{L}(n)$ by a few samples, because of the extra delay elements to the right of $\hat{E}^{\prime}\left(z^{2 M}\right)$. However, the transfer functions at the output of $\hat{\boldsymbol{E}}^{\prime}\left(z^{2 M}\right)$ have typically the same order. It is therefore not a serious loss of generality to assume that the degree of $\hat{E}^{\prime}(z)$ is equal to that of its $L$ th row.

\section{A Design Example}

Consider a three-channel QMF bank (i.e., $M=3$ ). We shall design a perfect-reconstruction system with real coefficient FIR analysis filters $H_{0}(z), H_{1}(z)$, and $H_{2}(z)$ of orders 55,52 , and 55 , respectively. The design will be based on the structure of Fig. 8. The first step is to design a 3rd band zero-phase filter $G_{1}(z)$ of order 104, with $G_{1}\left(e^{j \omega}\right)$ $\geq 0$ for all $\omega$, and obtain a spectral factor $H_{1}(z)$. This step can be simplified by noting that $G_{1}(z)$ has the form $G_{1}^{\prime}\left(-z^{2}\right)$ where $G_{1}^{\prime}(z)$ is a 3 rd band zero-phase low-pass filter of order 52 with $G_{1}^{\prime}\left(e^{j \omega}\right) \geq 0$. With $H_{1}^{\prime}(z)$ denoting a spectral factor of $G_{j}^{\prime}(z)$ ( so that $H_{1}^{\prime}(z)$ has order 26), we have $H_{1}(z)=H_{1}^{\prime}\left(-z^{2}\right)$.
In terms of the notations in Section II, let $H_{1}^{\prime}(z)$ be of the form $H_{1}^{\prime}(z)=H_{10}^{\prime}(z) H_{11}^{\prime}(z)$ where $H_{10}^{\prime}(z)$ has order $l_{0}$ and $H_{11}^{\prime}(z)$ has order $l_{1}$. Clearly, we have $l_{0}+l_{1}=26$ so that from (27a), $p_{1}=2$. Thus, from (27b) we obtain $2 l_{0}-l_{1}=-2$. These relations give the unique values $l_{0}$ $=8$, and $l_{1}=18$. We can now proceed to design the lowpass filter $H_{1}^{\prime}(z)$ of order 26 in the manner described in Section II, and obtain $H_{1}(z)=H_{1}^{\prime}\left(-z^{2}\right)$. The objective function analogous to (16) to be minimized is

$$
\int_{\pi / 3+\epsilon}^{\pi}\left|H_{10}^{\prime}\left(e^{j \omega}\right)\right|^{2}\left|H_{11}^{\prime}\left(e^{j \omega}\right)\right|^{2} d \omega .
$$

In the example, $\epsilon=0.2 \pi$ was used. Notice that the order of $G_{1}^{\prime}(z)$ is 52 , whereas that of $G_{10}^{\prime}(z)$ is only 16 . The spectral factorization of $G_{10}^{\prime}(z)$, which is required in the procedure of Section II, is therefore much simpler than a direct factorization of $G_{1}^{\prime}(z)$. Fig. 9 shows the response of the bandpass filter $G_{1}(z)$ and its spectral factor $H_{1}(z)$. Note that $G_{1}(z)$ has several zeros on the unit circle, and has about $140 \mathrm{~dB}$ stopband attenuation.

With the above $H_{1}(z)$ used as an "initial value" for the transfer function $H_{1}(z)$ in Fig. 8, the middle row $\left[\hat{\boldsymbol{e}}_{1}(z)\right]^{T}$ of the $3 \times 3$ matrix $\hat{\boldsymbol{E}}^{\prime}(z)$ is completely determined. We then synthesize $\hat{\boldsymbol{e}}_{1}(z)$ as $\hat{\boldsymbol{e}}_{1}(z)=\boldsymbol{U}(z) \boldsymbol{P}_{1}$, thereby initializing the vectors $\boldsymbol{u}_{k}$ and $\boldsymbol{P}_{1}$.

We can express $\left[\hat{\boldsymbol{E}}^{\prime}(z)\right]^{T}$ as $\left[\hat{\boldsymbol{E}}^{\prime}(z)\right]^{T}=\boldsymbol{U}(z) \boldsymbol{H}_{0}$ where the $3 \times 3$ matrix $\boldsymbol{H}_{0}$ is $\boldsymbol{H}_{0}=\left[\boldsymbol{H}_{00} \boldsymbol{P}_{1} \boldsymbol{H}_{02}\right]$. Thus, $\boldsymbol{H}_{0}$ is a real orthogonal matrix with middle column determined. It has only one extra degree of freedom (by Appendix D). Since $\hat{\boldsymbol{B}}$ is $1 \times 1$, it has no free parameters. (The matrices $\hat{\boldsymbol{B}}$ and $\boldsymbol{P}_{1}$ are not necessary for $M=3$.) In the example, the extra freedom in $\boldsymbol{H}_{0}$ was randomly initialized, and all the parameters [including the $\boldsymbol{u}_{k}$ vectors in $\hat{\boldsymbol{E}}^{\prime}(z)$ ] were reoptimized to minimize (2). Fig. 10 shows the complete lattice structure, where $V_{k}(z)$ are $3 \times 3$ degree-one lossless sections as in (33). The resulting analysis filter responses are shown in Fig. 11, whereas the impulse response coefficients are given in Table I. The convergence of the optimization was found to be much faster than our earlier techniques (in fact comparable to the design of a single-rate linear-phase lowpass filter of same order using [24]).

\section{Concluding Remarks}

The main purpose of this paper has been to provide an improved approach for designing $M$-band perfect-reconstruction FIR QMF banks based on lossless polyphase matrices. The improvements arise from judicious initialization of the analysis filters in the optimization process, more efficient characterization of FIR lossless systems by avoiding rotation operators, and finally the incorporation of symmetry between pairs of analysis filters. The improvements obtained are in terms of faster convergence of the optimization procedure, and in terms of better stopband attenuation of analysis filters for a given filter length and transition bandwidth. The techniques developed in this paper work for arbitrary $M$ (i.e., arbitrary number of channels). 


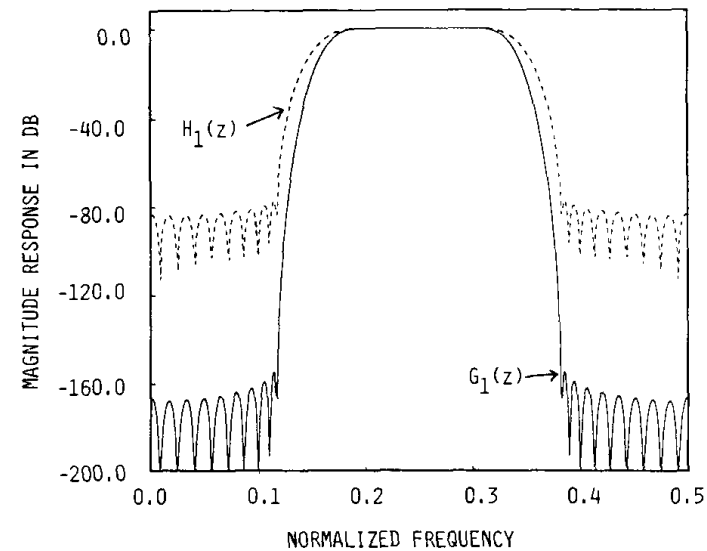

Fig. 9. The 3rd band filter $G_{1}(z)$ and its spectral factor $H_{1}(z)$.

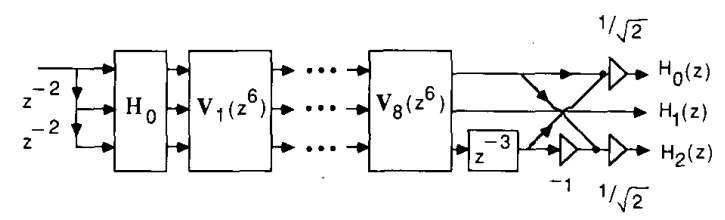

Fig. 10. The cascaded lossless structure for the analysis bank of the design example.

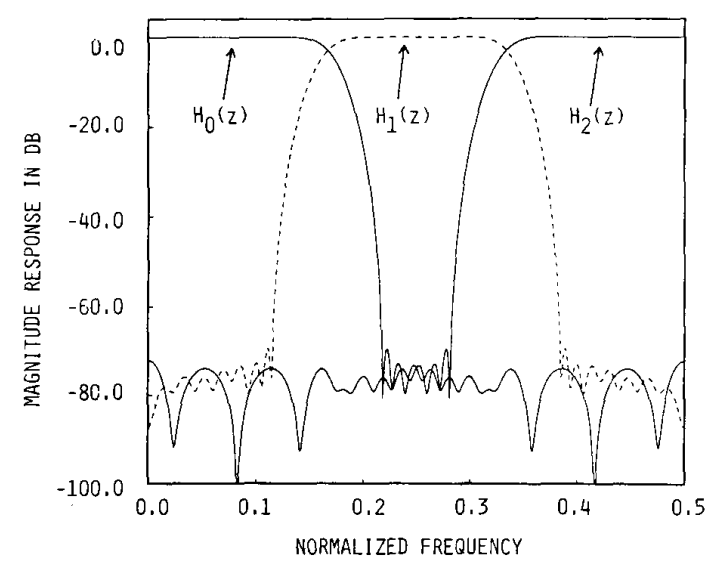

Fig. 11. Responses of the reoptimized analysis filters.

\section{APPENDIX A}

Losslessness of $\boldsymbol{E}(z)$ AND $M$ Th BAND PROPERTY OF $\tilde{H}_{k}(z) H_{k}(z)$

The analysis filter $H_{0}(z)$ can be written as

$$
H_{0}(z)=\left[\begin{array}{llll}
1 & z^{-1} & \cdots & z^{-(M-1)}
\end{array}\right] \boldsymbol{e}_{0}\left(z^{M}\right)
$$

where $\boldsymbol{e}_{0}^{T}(z)$ is the 0th row of $\boldsymbol{E}(z)$. By replacing $z$ with $z W^{-k}$ for $0 \leq k \leq M-1$, we can arrive at $M$ equations like (A.1). Stacking these together, we obtain

$$
\left[\begin{array}{l}
H_{0}(z) \\
H_{0}\left(z W^{-1}\right) \\
\vdots \\
H_{0}\left(z W^{-(M-1)}\right)
\end{array}\right]=\mathbf{W \Lambda}(z) e_{0}\left(z^{M}\right)
$$

where $\boldsymbol{W}$ is the $M \times M$ DFT matrix, and $\boldsymbol{\Lambda}(z)$ is a diagonal matrix of delays:

$$
\Lambda(z)=\left[\begin{array}{ccccc}
1 & 0 & 0 & \cdots & 0 \\
0 & z^{-1} & 0 & \cdots & 0 \\
\vdots & \vdots & \ddots & \vdots & \vdots \\
0 & 0 & 0 & \cdots & z^{-(M-1)}
\end{array}\right]
$$

Since $\boldsymbol{E}(z)$ is lossless, the vector $\boldsymbol{e}_{0}(z)$ is lossless. The matrices $\boldsymbol{W}$ and $\boldsymbol{\Lambda}(z)$ are clearly lossless. The product in (A.2) is therefore lossless (i.e., power complementary), so that

$$
\sum_{k=0}^{M-1} \tilde{H}_{0}\left(z W^{-k}\right) H_{0}\left(z W^{-k}\right)=c
$$

where $c$ is a nonzero constant. This proves that $G_{0}(z)$ $\triangleq \tilde{H}_{0}(z) H_{0}(z)$ is an $M$ th band filter. In other words, $H_{0}(z)$ is a spectral factor of an $M$ th band filter. The same is evidently true of $H_{k}(z), 1 \leq k \leq M-1$.

Conversely, we see that if $G_{0}(z)$ is an $M$ th band filter, then $H_{0}(z)$ satisfies (A.4), so that the left-hand side of (A.2) is lossless. It can then be verified that $\boldsymbol{e}_{0}(z)$ is lossless as well.

\section{APPENDIX B \\ Properties oF Diadics}

Let $v$ be an $M \times 1$ column vector, $v \neq 0$. Then $v v^{\dagger}$ is said to be a (Hermitian-)diadic. Some important properties of diadics are listed below.

1) The rank of such a matrix is unity ( since all the columns are scalar multiples of $v$ ) so that $M-1$ of the $M$ eigenvalues are equal to zero. The only nonzero eigenvalue is equal to $\boldsymbol{v}^{\dagger} \boldsymbol{v}$, with corresponding eigenvector equal to $\boldsymbol{v}$ (proof: $\boldsymbol{v} \boldsymbol{v}^{\dagger} \boldsymbol{v}=\boldsymbol{v}^{\dagger} \boldsymbol{v} \boldsymbol{v}$ by associativity).

2) Let $\boldsymbol{v}$ be of unit norm so that $\boldsymbol{v}^{\dagger} \boldsymbol{v}=1$. Then the matrix $\boldsymbol{I}-\boldsymbol{v} \boldsymbol{v}^{\dagger}$ has rank $M-1$. The only nonnull $\boldsymbol{w}$ such that $\left[I-v v^{\dagger}\right] w=0$ has the form $w=c v$, where $c \neq$ 0 is a scalar.

3) Consider a matrix of the form $A=I-2 v v^{\dagger}$, with $\boldsymbol{v} \neq \mathbf{0}$. Assume that $\boldsymbol{v}$ is such that $\boldsymbol{A}$ is unitary. Thus, $\boldsymbol{A}$ is unitary and Hermitian so that its eigenvalues are \pm 1 . Since $1-2 v^{\dagger} v$ is an eigenvalue, it should equal \pm 1 . With $v \neq 0$, this eigenvalue must be -1 , so that $\boldsymbol{v}^{\dagger} \boldsymbol{v}=$ 1. In summary, if a matrix of the form $I-2 v v^{\dagger}$ is unitary where $v$ is a column vector, then $v$ has unit norm.

4) Let $\boldsymbol{u}$ and $v$ be unit-norm vectors such that

$$
\boldsymbol{u}^{\dagger} \boldsymbol{v} \neq 0
$$

Consider the matrix

$$
A=\left[I-v v^{\dagger}\right]\left[I-u u^{\dagger}\right] .
$$

Clearly, $\boldsymbol{A} \boldsymbol{u}=\mathbf{0}$ by property 2 . It turns out that any nonnull vector $\boldsymbol{w}$ for which

$$
A w=0
$$

is such that

$$
\boldsymbol{w}=c \boldsymbol{u}, \quad c \neq 0 .
$$


TABLE I

ImpUlse Response CoEfFicients of The Reoptimized ANALYSis Filters in the Design EXample

\begin{tabular}{|c|c|c|c|}
\hline$m$ & Coefficients $h_{0}(n)$ & Coefficients $h_{1}(n)$ & Coefficients $h_{2}(n)$ \\
\hline 0 & $-1.4128229067239 \times 10^{-3}$ & $1.5056378297974 \times 10^{-5}$ & $-1.4128229067239 \times 10^{-3}$ \\
\hline$\overline{1}$ & 0 . & 0. & 0. \\
\hline 2 & $2.1414058710181 \times 10^{-2}$ & $6.6399724053250 \times 10^{-5}$ & $2.1414058710181 \times 10-2$ \\
\hline 3 & $9.1308218484911 \times 10^{-2}$ & 0. & $-9.1308218484911 \times 10^{-2}$ \\
\hline$\overline{4}$ & $2.2641530391958 \times 10^{-1}$ & $8.8793526437529 \times 10^{-5}$ & $2.2641530391958 \times 10^{-1}$ \\
\hline 5 & $3.9757826027754 \times 10^{-1}$ & 0. & $-3.9757826027754 \times 10^{-1}$ \\
\hline 6 & $5.1966245198362 \times 10^{-1}$ & $-1.6061927086436 \times 10^{-4}$ & $5.1966245198362 \times 10^{-1}$ \\
\hline$\overline{7}$ & $4.9519441407873 \times 10^{-1}$ & 0. & $-4.9519441407873 \times 10^{-1}$ \\
\hline 8 & $2.9453172543002 \times 10^{-1}$ & -5.9188010394438 & $2.9453172543002 \times 10^{-1}$ \\
\hline 9 & $5.2368950426968 \times 10^{-3}$ & 0. & $-5.2368950426968 \times 10^{-3}$ \\
\hline 10 & $-2.0994092811453 \times 10^{-1}$ & $8.2758834028361 \times 10^{-4}$ & $-2.0994092811453 \times 10^{-1}$ \\
\hline 11 & $-2.3111384039953 \times 10^{-1}$ & 0. & $2.3111384039953 \times 10^{-1}$ \\
\hline 12 & $-7.8847322029914 \times 10^{-2}$ & $375 \times 10^{-3}$ & $-7.8847322029914 \times 10^{-2}$ \\
\hline 13 & $1.0104466433718 \times 10^{-1}$ & 0. & $-1.0104466433718 \times 10^{-1}$ \\
\hline 14 & $1.6112218318323 \times 10^{-1}$ & $109176 \times 10^{-3}$ & $1.6112218318323 \times 10^{-1}$ \\
\hline 15 & $7.4057850344872 \times 10^{-2}$ & 0. & $-7.4057850344872 \times 10^{-2}$ \\
\hline 16 & $-5.8686064368913 \times 10^{-2}$ & $-1.7834882160760 \times 10^{-3}$ & $-5.8686064368913 \times 10^{-2}$ \\
\hline 17 & $-1.1225434647491 \times 10^{-1}$ & 0. & $1.1225434647491 \times 10^{-1}$ \\
\hline 18 & $-5.4550575653918 \times 10^{-2}$ & $45031 \times 10^{-2}$ & $-5.4550575653918 \times 10^{-2}$ \\
\hline 19 & $4.0281521826881 \times 10^{-2}$ & 0. & $-4.0281521826881 \times 10^{-2}$ \\
\hline 20 & $7.7614334886641 \times 10^{-2}$ & $727 \times 10^{-2}$ & $7.7614334886641 \times 10^{-2}$ \\
\hline 21 & $3.4995958094679 \times 10^{-2}$ & 0. & $-3.4995958094679 \times 10^{-2}$ \\
\hline 22 & -3.037697 & $599 \times 10^{-2}$ & $-3.0376976569541 \times 10^{-2}$ \\
\hline$\overline{23}$ & $-5.1824955200610 \times 10^{-2}$ & 0. & $5.1824955200610 \times 10^{-2}$ \\
\hline 24 & $-1.9388392668025 \times 10^{-2}$ & $4.7813912371864 \times 10^{-2}$ & -1.9388392668 \\
\hline 25 & $2.3116098694287 \times 10^{-2}$ & 0. & $-2.3116098694287 \times 10^{-2}$ \\
\hline 26 & $21 \times 10^{-2}$ & $-1.0294204447447 \times 10^{-2}$ & $3.2571783564021 \times 10^{-2}$ \\
\hline 27 & $8.5801203815075 \times 10^{-3}$ & 0. & $-8.5801203815075 \times 10^{-3}$ \\
\hline 28 & $-1.6753271999023 \times 10^{-2}$ & -7.7918425 & -1.675327199 \\
\hline 29 & $-1.8667164769370 \times 10^{-2}$ & 0. & $1.8667164769370 \times 10^{-2}$ \\
\hline 30 & $-2.2382174791812 \times 10^{-3}$ & $1.0751032541568 \times 10^{-1}$ & -2.238217479 \\
\hline 31 & $841 \times 10^{-2}$ & 0. & $-1.109651911384 \overline{10^{-2}}$ \\
\hline 32 & $9.4068169151632 \times 10^{-3}$ & $6.7499047357221 \times 10^{-3}$ & $9.4068169151632 \times 10^{-3}$ \\
\hline 33 & $-6.9083772961599 \times 10^{-4}$ & $\overrightarrow{0 .}$ & $6.9083772961599 \times 10^{-4}$ \\
\hline 34 & $-6.5014160081924 \times 10^{-3}$ & $-1.8443792714387 \times 10^{-1}$ & $-6.5014160081924 \times 10^{-3}$ \\
\hline 35 & $-3.8902922683508 \times \overline{10^{-3}}$ & $\overline{0 .}$ & $3.8902922683508 \times 10^{-3}$ \\
\hline$\overline{36}$ & $1.4380900534430 \times 10^{-3}$ & $2.2127681299343 \times 10^{-1}$ & $1.4380900534430 \times 10^{-3}$ \\
\hline 37 & $3.2229563292928 \times 10^{-3}$ & 0. & $-3.2229563292928 \times 10^{-3}$ \\
\hline 38 & $1.1229696945844 \times 10^{-3}$ & $-6.1951636758746 \times 10^{-3}$ & $1.1229696945844 \times 10^{-3}$ \\
\hline 39 & $-1.1471284909200 \times 10^{-3}$ & 0. & $1.1471284909200 \times 10^{-3}$ \\
\hline 40 & $-1.2482566010628 \times 10^{-3}$ & $-3.3826132198080 \times 10^{-1}$ & $-1.2482566010628 \times 10^{-3}$ \\
\hline 41 & $-5.8375452585888 \times 10^{-5}$ & 0. & $5.8375452585888 \times 10^{-5}$ \\
\hline 42 & $5.9489610310688 \times 10^{-4}$ & $5.6761980190258 \times 10^{-1}$ & $5.9489610310688 \times 10^{-4}$ \\
\hline 43 & $3.0406128784832 \times 10^{-4}$ & 0. & $-3.0406128784832 \times 10^{-4}$ \\
\hline 44 & $-1.5404701840277 \times 10^{-4}$ & $-5.5034960832577 \times 10^{-1}$ & $-1.5404701840277 \times 10^{-4}$ \\
\hline 45 & $-1.9182526916816 \times 10^{-4}$ & 0. & $1.9182526916816 \times 10^{-4}$ \\
\hline 46 & $1.3642678226151 \times 10^{-5}$ & $3.5985541240956 \times 10^{-1}$ & $1.3642678226151 \times 10^{-5}$ \\
\hline 47 & $8.4501036966324 \times 10^{-5}$ & 0. & $-8.4501036966324 \times 10^{-5}$ \\
\hline 48 & $2.4216033174065 \times 10^{-6}$ & $-1.5882736075304 \times 10^{-1}$ & $2.4216033174065 \times 10^{-6}$ \\
\hline 49 & $-3.4346594598878 \times 10^{-5}$ & 0. & $3.4346594598878 \times 10^{-5}$ \\
\hline 50 & $-6.5174428259106 \times 10^{-7}$ & $4.2746400100218 \times 10^{-2}$ & $-6.5174428259106 \times 10^{-7}$ \\
\hline 51 & $1.3132483913474 \times 10^{-5}$ & 0. & $-1.3132483913474 \times 10^{-5}$ \\
\hline 52 & $7.6751821402677 \times 10^{-8}$ & $-5.0339744493869 \times 10^{-3}$ & $7.6751821402677 \times 10^{-8}$ \\
\hline 53 & $-3.5344439963839 \times 10^{-6}$ & 0. & $3.5344439963839 \times 10^{-6}$ \\
\hline 54 & 0 . & 0 . & 0. \\
\hline 55 & $4.1622921997806 \times 10^{-7}$ & 0. & $-4.1622921997806 \times 10^{-7}$ \\
\hline
\end{tabular}

Property 4 implies that $\boldsymbol{A}$ has rank $M-1$. To prove property 4 , note that (A.7) implies

$$
\left[I-u u^{\dagger}\right] w=v v^{\dagger}\left[I-u u^{\dagger}\right] w
$$

which shows that

$$
\left[I-u u^{\dagger}\right] w
$$

is either an eigenvector of $\boldsymbol{v} \boldsymbol{v}^{\dagger}$ (with unit eigenvalue) or the null vector. If the former is true then

$$
\left[I-u u^{\dagger}\right] w=c v
$$

by Property 1. However, (A.11) implies $\boldsymbol{u}^{\dagger} \boldsymbol{v}=\mathbf{0}$ violating (A.5) unless $c=0$. The vector (A.10) is thus null, proving, by Property 2 , that $w$ has the form (A.8). 


\section{APPENDIX C \\ ON THE RANK OF $\boldsymbol{h}_{N-1}(0)$}

Let $\boldsymbol{H}_{N-1}(z)$ be an $M \times M$ lossless system of degree $N$ -1 given by $\boldsymbol{H}_{N-1}(z)=\Sigma_{n=0}^{K} \boldsymbol{h}_{N-1}(n) z^{-n}$. We then have $K \leq N-1$. We know from Property 3.2 that $\boldsymbol{h}(0)$ is singular. If there exists a column of $\boldsymbol{H}_{N-1}(z)$, denoted $\boldsymbol{P}_{N-1}(z)$, such that its degree is $N-1$, then at least one column of $\boldsymbol{h}_{N-1}(N-1)$ is nonzero, so that $K=N-1$. This means that in the factorization (32) with $V_{k}(z)$ as in (33), the product

$$
\boldsymbol{v}_{N-1} \boldsymbol{v}_{N-1}^{\dagger} \boldsymbol{v}_{N-2} \boldsymbol{v}_{N-2}^{\dagger} \cdots \boldsymbol{v}_{1} \boldsymbol{v}_{1}^{\dagger}
$$

is a nonnull vector. This in turn implies the following:

$$
\boldsymbol{v}_{k}^{\dagger} \boldsymbol{v}_{k-1} \neq 0, \quad 2 \leq k \leq N-1
$$

Now consider the expression for $\boldsymbol{h}_{N-1}(0)$ in terms of the representation (32)

$$
\begin{aligned}
\boldsymbol{h}_{N-1}(0)= & {\left[\boldsymbol{I}-\boldsymbol{v}_{N-1} \boldsymbol{v}_{N-1}^{\dagger}\right]\left[\boldsymbol{I}-\boldsymbol{v}_{N-2} \boldsymbol{v}_{N-2}^{\dagger}\right] } \\
& \cdots\left[\boldsymbol{I}-\boldsymbol{v}_{1} \boldsymbol{v}_{1}^{\dagger}\right] \boldsymbol{H}_{0} .
\end{aligned}
$$

Since $\boldsymbol{H}_{0}$ is unitary, the rank of $\boldsymbol{h}_{N-1}(0)$ is equal to that of $\boldsymbol{h}_{N-1}(0) \boldsymbol{H}_{0}^{\dagger}$. It turns out that under the condition (A.13), this rank is precisely equal to $M-1$. This can be proved by repeated application of the argument given in Property 4 of Appendix B.

\section{APPENDIX D \\ EXPRESSIONS FOR $N_{f}$ AND $N_{f}^{\prime}$}

Let $\boldsymbol{U}=\left[\begin{array}{llll}\boldsymbol{u}_{0} & \boldsymbol{u}_{1} & \cdots & \boldsymbol{u}_{M-1}\end{array}\right]$ be an $M \times M$ unitary matrix. The unitary property can be expressed in terms of the $\left(\begin{array}{c}M \\ 2\end{array}\right)$ constraints

$$
\begin{array}{r}
\boldsymbol{u}_{k}^{\dagger} \boldsymbol{u}_{l}=0, \quad 0 \leq k \leq M-1, \\
k+1 \leq l \leq M-1
\end{array}
$$

and the $M$ constraints

$$
\boldsymbol{u}_{k}^{\dagger} \boldsymbol{u}_{k}=1, \quad 0 \leq k \leq M-1 .
$$

The left-hand sides of each of the ( $\left(\begin{array}{c}M \\ 2\end{array}\right)$ constraints in (A.15) are, in general, complex, whereas those in (A.16) are real. Altogether, we therefore have a total of $2\left(\begin{array}{c}M \\ 2\end{array}\right)+M$ real constraints because of (A.15) and (A.16). An arbitrary $M$ $\times M$ matrix with complex entries has $2 M^{2}$ real-valued degrees of freedom. Subtracting the $2\left(\begin{array}{c}M \\ 2\end{array}\right)+M$ constraints due to unitariness, we arrive at the number of degrees of freedom [21] $N_{\text {unitary }}=M^{2}$ for an $M \times M$ unitary matrix. A similar calculation with real matrices shows that the number of real-valued degrees of freedom for an $M \times$ $M$ real orthogonal matrix is equal to $\left(\begin{array}{c}M \\ 2\end{array}\right)$. These freedoms are commonly expressed as planar rotation angles [21], [18].

Now assume that one particular column, say $\boldsymbol{u}_{0}$, of the $M \times M$ unitary matrix $U$ is fixed. In the complex case, this takes away $2 M-1$ real-valued degrees of freedom (because the column has $M$ complex entries constrained by $\left.\boldsymbol{u}_{0}^{\dagger} \boldsymbol{u}_{0}=1\right)$. This leaves behind $N_{f}^{\prime}=M^{2}-2 M+1$ freedoms which is (55). For the case of real matrices, a similar calculation shows that the number of degrees of freedom in a real orthogonal matrix with a fixed column is equal to $N_{f}=\left(\begin{array}{c}M \\ 2\end{array}\right)-(M-1)$ which is (8).

\section{REFERENCES}

[1] A. Croisier, D. Esteban, and C. Galand, "Perfect channel splitting by use of interpolation/decimation/tree decomposition techniques," presented at the Int. Symp. Info., Circuits, and Syst., Patras, Greece, 1976.

[2] R. E. Crochiere and L. R. Rabiner, Multirate Digital Signal Processing. Englewood Cliffs, NJ: Prentice-Hall, 1983.

[3] V. K. Jain and R. E. Crochiere, "Quadrature mirror filter design in the time domain," IEEE Trans. Acoust., Speech, Signal Processing, vol. ASSP-32, pp. 353-361, Apr. 1984.

[4] C. R. Galand and H. J. Nussbaumer, "New quadrature mirror filter structures,"' IEEE Trans. Acoust., Speech, Signal Processing, vol. ASSP-32, pp. 522-531, June 1984.

[5] M. J. T. Smith and T. P. Barnwell, III, "A new filter bank theory for time-frequency representation," IEEE Trans. Acoust., Speech, Signal Processing, vol. ASSP-35, pp. 314-327, Mar. 1987.

[6] - - " "Exact reconstruction techniques for tree-structured subband coders," IEEE Trans. Acoust., Speech, Signal Processing, vol. ASSP34 , pp. 434-441, June 1986.

[7] J. H. Rothweiler, "Polyphase quadrature filters, a new sub-band coding technique,"' in Proc. IEEE Int. Conf. ASSP, Boston, MA, Apr. 1983, pp. 1980-1983.

[8] P. L. Chu, "Quadrature mirror filter design for an arbitrary number of equal bandwidth channels," IEEE Trans. Acoust., Speech, Signal Processing, vol. ASSP-33, pp. 203-218, Feb. 1985.

[9] M. Vetterli, "A theory of multirate filter banks," IEEE Trans. Acoust., Speech, Signal Processing, vol. ASSP-35, pp. 356-372, Mar. 1987.

[10] - "Splitting a signal into sub-sampled channels allowing perfect reconstruction," in Proc. IASTED Conf. Appl. Signal Processing Digital Filtering, Paris, France, June 1985.

[11] K. Swaminathan and P. P. Vaidyanathan, "Theory and design of uniform DFT, parallel, quadrature mirror filter banks," IEEE Trans. Circuits Syst., vol. CAS-33, pp. 1170-1191, Dec. 1986.

[12] P. P. Vaidyanathan, "Theory and design of $M$-channel maximally decimated quadrature mirror filters with arbitrary $M$, having the perfect-reconstruction property," IEEE Trans. Acoust., Speech, Signal Processing, vol. ASSP-35, pp. 476-492, Apr. 1987.

[13] _ " "Quadrature mirror filter banks, $M$-band extensions and perfectreconstruction techniques," IEEE ASSP Mag., pp. 4-20, July 1987.

[14] M. Bellanger, G. Bonnerot, and M. Coudreuse, "Digital filtering by polyphase network: Application to sample rate alteration and filter banks," IEEE Trans. Acoust., Speech, Signal Processing, vol. ASSP24, pp. 109-114, Apr. 1976.

[15] T. Q. Nguyen and P. P. Vaidyanathan, "Maximally decimated perfect-reconstruction FIR filter banks with pairwise mirror-image analysis (and synthesis) frequency responses," IEEE Trans. Acoust., Speech, Signal Processing, vol. 36, pp. 693-706, May 1988.

[16] P. P. Vaidyanathan, T. Q. Nguyen, and T. Saramäki, "Improved approach for design of perfect reconstruction FIR QMF banks, with lossless lattice structures,' in Proc. IEEE Int. Conf. ASSP, New York, Apr. 1988, pp. 1471-1474.

[17] P. P. Vaidyanathan, Z. Doğanata, and T. Q. Nguyen, "More results on the perfect reconstruction problem in $M$-band parallel QMF bank," in Proc. IEEE Int. Symp. Circuits Syst,., Philadelphia, May 1987, pp. $847-850$.

[18] Z. Doğanata, P. P. Vaidyanathan, and T. Q. Nguyen. “General synthesis procedures for FIR lossless transfer matrices for perfect-reconstruction multirate filter bank applications," IEEE Trans. Acoust., Speech, Signal Processing, vol. 36, pp. 1561-1574, Oct. 1988.

[19] P. P. Vaidyanathan, "A tutorial on multirate digital filter banks," in Proc. IEEE Int. Symp. Circuits Syst., Espoo, Finland, June 1988, pp. 2241-2248.

[20] T. Q. Nguyen, T. Saramäki, and P. P. Vaidyanathan, "Eigenfilters for the design of special transfer functions with applications in multirate signal processing," in Proc. IEEE Int. Conf. ASSP. New York, Apr. 1988, pp. 1467-1470.

[21] F. D. Murnaghan, The Unitary and Rotation Groups. Washington, DC: Spartan Books, 1962.

[22] T. Kailath, Linear Systems. Englewood Cliffs, NJ: Prentice-Hall, 1980

[23] F. Mintzer, "On half-band, third band and $N$ th band FIR filters and their design." IEEE Trans. Acoust., Speech, Signal Processing, vol. ASSP-30. pp. 734-738, Oct. 1982. 
[24] J. H. McClellan and T. W. Parks, "A unified approach to the design of optimum FIR linear phase digital filters," IEEE Trans. Circuit Theory, vol. CT-20, pp. 697-701, Nov. 1973.

[25] G. A. Mian and A. P. Nainer, "A fast procedure to design equiripple minimum-phase FIR filters," IEEE Trans. Circuits Syst., vol. CAS29, pp. 327-331, May 1982.

[26] L. R. Rabiner and B. Gold, Theory and Application of Digital Signal Processing. Englewood Cliffs, NJ: Prentice-Hall, 1975.

[27] P. P. Vaidyanathan, "Design and implementation of digital FIR filters," in Handbook on Digital Signal Processing, D. F. Elliott, Ed. New York: Academic, 1987. pp. 55-172.

[28] P. P. Vaidyanathan and T. Q. Nguyen, “Eigenfilters: A new approach to least squares FIR filter design and applications including Nyquist filters," IEEE Trans. Circuits Systems, vol. CAS-34, pp 11-23, Jan. 1987.

[29] V. Belevitch, Classical Network Theory. San Francisco, CA: Holden-Day, 1968.

[30] B. D. O. Anderson and S. Vongpanitlerd, Network Analysis and Synthesis. Englewood Cliffs. NJ: Prentice-Hall, 1973

[31] T. H. Ramstad, "Analysis/synthesis filter banks with critical sampling," presented at the Int. Conf. Digital Signal Processing, Florence, Sept. 1984

[32] T. Saramäki and Y. Neuvo, “A class of FIR Nyquist $(N$ th band) filters with zero intersymbol interference," IEEE Trans. Circuits Syst., vol. CAS-34, pp. 1182-1190, Oct. 1987.

P. P. Vaidyanathan (S'80-M'83-SM' 88), for a photograph and biography, see p. 690 of the May 1989 issue of this Transactions.

Truong Q. Nguyen (S'86), for a photograph and biography, see p. 690 of the May 1989 issue of this Transactions.

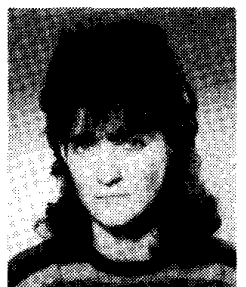

Zinnur Doganata ( $\mathrm{S}^{*} 86$ ) was born in Ankara. Turkey, on October 29.1959. She received the B.S. and M.S. degrees in electrical engineering from the Middle East Technical University, Ankara, in 1981 and 1984 , respectively.

During the period 1981-1984 she worked part time for the Military Electronic Industries of Turkey. Presently she is pursuing the Ph.D. degree in electrical engineering at the California Institute of Technology. Pasadena. Her main research in terests are in digital signal processing and lossless systems.

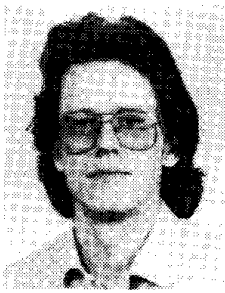

Tapio Saramäki was born in Orivesi, Finland, on June 12, 1953. He received the degrees of Diploma Engineer (with honors) and Doctor of Technology (with honors) in electrical engineering from the Tampere University of Technology. Tampere, Finland, in 1978 and 1981, respectively.

Since 1977 he has been with the Department of Electrical Engineering of the Tampere University of Technology. From 1979 to 1981 he served as a Research Assistant, and from 1982 to 1986 as a Research Fellow, both financed by the Academy of Finland. Since 1987 he has held various research and teaching positions at the Tampere University of Technology. Currently he is an Associate Professor of Electrical Engineering. In 1982, 1985, and 1986, he was a Visiting Research Scholar at the University of California. Santa Barbara. He has authored more than 70 international journal and conference articles. His research interests are in the areas of digital signal processing and approximation theory.

Dr. Saramäki received the 1987 Guillemin-Cauer Award for the best paper of the IEEE TRANSACTIONS ON CiRCUITS AND SySTEMS. He is a founding member of the Median-Free Group International. 